\title{
Bulk fatigue induced by surface reconstruction in layered Ni-rich oxide cathodes for Li-
} ion batteries

Chao Xu, ${ }^{1,2}$ Katharina Märker, ${ }^{1,2}$ Juhan Lee, ${ }^{2,3}$ Amoghavarsha Mahadevegowda, ${ }^{2,4}$ Philip J. Reeves, ${ }^{1,2}$ Sarah Day, ${ }^{5}$ Matthias F. Groh, ${ }^{1}$ Steffen Emge, ${ }^{1}$ Caterina Ducati, ${ }^{2,4}$ B. Layla Mehdi, ${ }^{2,3}$ Chiu Tang, ${ }^{5}$ Clare P. Grey ${ }^{*}, 1,2$

${ }^{1}$ Department of Chemistry, University of Cambridge, Lensfield Road, Cambridge CB2 1EW, United Kingdom

${ }^{2}$ The Faraday Institution, Quad One, Harwell Science and Innovation Campus, Didcot OX11 ORA, United Kingdom

${ }^{3}$ Department of Mechanical, Materials and Aerospace Engineering, University of Liverpool, Liverpool, L69 3GH, United Kingdom

4 Department of Materials Science and Metallurgy, University of Cambridge, 27 Charles Babbage Road, Cambridge CB3 OFS, United Kingdom

${ }^{5}$ Diamond Light Source, Harwell Science and Innovation Campus, Didcot, UK

\begin{abstract}
Ni-rich layered cathode materials are among the most promising candidates for high energy density Li-ion batteries. However, the low cobalt containing materials suffer from rapid degradation, the underlying mechanism of which is still poorly understood. We herein report a novel structure-drive degradation mechanism for the NMC811( $\left.\mathrm{LiNi}_{0.8} \mathrm{Mn}_{0.1} \mathrm{Co}_{0.1} \mathrm{O}_{2}\right)$ cathode, in which a proportion of the material exhibits a lowered accessible state-of-charge (SoC) at the end of charge after repetitive cycling, i.e. becomes fatigued. Ex-situ and operando longduration high-resolution X-ray diffraction enabled by a laser-thinned coin cell design clearly shows the emergence of the fatigued phase and the increase in its population as the cycling progresses. We show that the fatigue degradation is a structure-driven process rather than originating solely due to kinetic limitations or inter-granular cracking. No bulk phase transformations or increase in $\mathrm{Li} / \mathrm{Ni}$ antisite mixing were observed by diffraction; no significant change in the local structure or Li-ion mobility of the bulk were observed by ${ }^{7} \mathrm{Li}$ solid-state NMR spectroscopy. Instead, we propose that the fatigue process is a result of the high interfacial lattice strain between the reconstructed surface and the bulk layered structure when the latter is at SoCs above a distinct threshold of $\sim 75 \%$. This mechanism is expected to be universal to Ni-rich layer cathodes, and our findings provide a fundamental guide for designing effective approaches to mitigate such deleterious processes.
\end{abstract}




\section{Introduction}

Rapid advancements in the performance of Li-ion batteries (LIBs) have enabled the commercial deployment of electric vehicles (EVs), which is a crucial step forward to reduce fossil fuel consumption and develop a more sustainable society. ${ }^{1,2}$ High Ni-content layered Li transition metal oxides, for instance, $\mathrm{LiNi}_{0.8} \mathrm{Co}_{0.15} \mathrm{Al}_{0.05} \mathrm{O}_{2}$ (NCA) and $\mathrm{LiNi}_{\mathrm{x}} \mathrm{Mn}_{\mathrm{y}} \mathrm{Co}_{(1-\mathrm{x}-\mathrm{y})} \mathrm{O}_{2}$ (NMC, $\mathrm{x} \geq 0.5$ ) are currently used as EV LIB cathode materials owing to their superior energy and power densities. ${ }^{2-5}$ Among all the Ni-rich cathodes, $\mathrm{LiNi}_{0.8} \mathrm{Mn}_{0.1} \mathrm{Co}_{0.1} \mathrm{O}_{2}$, denoted as NMC811, is particularly attractive due to its high specific capacity and potentially lower materials cost and environmental impact arising from its lower cobalt content. ${ }^{3,4}$

Despite all its merits, NMC811 and Ni-rich layered materials, suffer from poor cycle life compared to their lower Ni-content analogues. ${ }^{6-9}$ Significant efforts have been devoted to unravelling degradation mechanisms of Ni-rich cathodes, and several potential origins have been proposed. First, Ni-rich cathode materials are susceptible to chemo-mechanical degradation, such as inter-granular cracking under deep cycling (i.e., high upper cut-off voltages). This is largely attributed to the strong anisotropic evolution of the crystal structure during Li extraction/insertion, and particularly the rapid lattice collapse at high states of charge (SoCs). ${ }^{10}$ Specifically, as Li is being removed from the NMC on charge, the unit cell (isostructure of $\alpha-\mathrm{NaFeO}_{2}$ with space group $R \overline{3} \mathrm{~m}$ ) gradually contracts along the $a / b$ direction while expanding along the $\mathrm{c}$ direction initially, and then collapses rapidly from $\sim 70 \% \mathrm{Li}$ removal and onwards. ${ }^{11,12}$ Second, the surface of Ni-rich materials undergoes oxygen loss and concomitant structural transformation from the initially layered, to spinel and/or rock saltlike, ${ }^{13-15}$ more densified structures such as $\mathrm{Ni}_{0.25} \mathrm{NiO}_{2}, \mathrm{Ni}_{0.5} \mathrm{NiO}_{2},{ }^{16}$ following electrochemical cycling. Evidence for these transformations even following contact with battery electrolytes has also emerged. ${ }^{14,16}$ Such reconstructed structures are poorer Li-ion and electrical conductors which are thought to impede lithiation and delithiation, and consequently deteriorate battery performance. ${ }^{14,17}$ Third, more severe electrolyte degradation occurs on higher Ni-content NMC materials. ${ }^{7,18,19}$ Jung et al. systematically studied a series of NMC cathode materials and showed that NMC811 has a significantly lower onset voltage for lattice oxygen evolution and more gas evolution at a lower voltage arising from of electrolyte decomposition compared to $\mathrm{LiNi}_{1 / 3} \mathrm{Mn}_{1 / 3} \mathrm{Co}_{1 / 3} \mathrm{O}_{2}$ and $\mathrm{LiNi}_{0.6} \mathrm{Mn}_{0.2} \mathrm{Co}_{0.2} \mathrm{O}_{2}{ }^{7,18}$

Although the surface reconstruction of Ni-rich layer cathodes is widely acknowledged, no major structural transformations in the bulk of these cathode materials have been observed. For example, NMC811 does not show any clear evidence for the $\mathrm{O} 3$ to $\mathrm{O} 1$ phase transition $(\mathrm{O} 3 / \mathrm{O} 1$ corresponding to the Delmas annotation ${ }^{20}$; also known as the $\mathrm{H} 2-\mathrm{H} 3$ transition, $\mathrm{H}$ denoting the formation of a hexagonal phase). ${ }^{11,12}$ This is a detrimental process known to occur in its analogous material $\mathrm{LiNiO}_{2}$ at highly delithiated states. ${ }^{21-23}$ Even after extended cycling, the long-range structure remains robust. ${ }^{9,24} \mathrm{Li}$ et al. performed a comprehensive investigation on the long-term cyclability of NCA and $\mathrm{LiNi}_{0.7} \mathrm{Mn}_{0.15} \mathrm{Co}_{0.15} \mathrm{O}_{2}$, and observed only a slight linebroadening in diffraction peaks and minor increase in anti-site cation disorder ( $\mathrm{Ni}$ at Li sites) with no appearance of new crystallographic phases. ${ }^{24}$ By contrast, Kleiner et al. observed the 
presence of multiple rhombohedral NCA phases which have the same symmetry but noticeably difference lattice constants in the fatigued cathode electrode after extensive electrochemical ageing. ${ }^{25}$ The authors identified that one of these rhombohedral phases is no longer electrochemically active, and it remains nearly fully lithiated when the electrode is charged to high potentials. ${ }^{25}$ Later, Liu et al. observed phase segregation in NCA at much earlier ageing stage ( $92^{\text {nd }}$ cycle), and identified two distinct NCA populations that react differently during cycling: the "active" population behaves similarly to fresh material while the "sluggish" portion shows a reduced active $\mathrm{SoC}$ range. ${ }^{26}$ The authors attributed the formation of the "sluggish" NCA population to the loss of connectivity of subsurface grains as a result of intergranular fracturing of polycrystalline aggregates. More recently, Schweidler et al. reported similar observations in Ni-rich $\mathrm{NMC}\left(\mathrm{Li}_{1+\mathrm{x}}\left(\mathrm{Ni}_{0.85} \mathrm{Co}_{0.1} \mathrm{Mn}_{0.05}\right)_{1-\mathrm{x}} \mathrm{O}_{2}, \mathrm{NCM} 851005\right)$, identifying the presence of a more lithiated phase at high SoCs. Moreover, its phase-fraction gradually increases as the battery ages, and the authors proposed a different mechanism involving an increase in the kinetic limitations of the fatigued cathode. To date, no clear-cut explanation as to what controls whether the cathode material becomes only partially reactive or completely inactive after electrochemical ageing has emerged, and the underlying mechanisms must be clarified so as to provide a rational basis for mitigating this degradation mechanism.

In this work, we used a variety of characterisation techniques to identify the origin of the fatigue process of NMC811 cathodes. A novel coin-cell with laser-thinned X-ray window was utilised for operando long-duration X-ray diffraction to study the fatigue process during electrochemical ageing. This cell design is fundamentally different from the conventional in situ powder X-ray diffraction (PXRD) coin cells which use open casings and are sealed with Kapton tapes. Such cells are known to be susceptible to air leakage and therefore cannot be used for long-term ageing studies. We first confirmed that the NMC811 cathode becomes fatigued after electrochemically ageing and shows the co-existence of particles at multiple SoCs using high-resolution synchrotron X-ray diffraction. ${ }^{7} \mathrm{Li}$ solid-state nuclear magnetic resonance spectroscopy, ssNMR, was then used to investigate the change in Li local structure and mobility after ageing. We then examined the two hypothesises in literature, namely slow kinetics and inter-granular cracking, by charging electrochemically aged polycrystalline as well as single-crystal cathodes using an extremely slow rate and extended upper cut-off voltages. The experimental results show the fatigue process is not a result of intergranular-cracking, kinetic limitations or a decrease in bulk Li-ion mobility. Instead, we propose that it is due to the high interfacial lattice strain between the bulk layered structure and rock salt surface reconstruction layer - seen in TEM micrographs - when the NMC cathode is at high SoCs. 


\section{Experimental}

Materials. Polycrystalline NMC811 and graphite electrodes are fabricated and kindly provided by the Argonne National Laboratory CAMP facility, and matched for $4.2 \mathrm{~V}$ full-cell cycling (details can be found in the previous report). ${ }^{12}$ Briefly, the NMC cathode (batch code A-C020) consists of $90 \mathrm{wt} \%$ NMC811 (Targray), $5 \mathrm{wt} \%$ PVDF binder (Solvay 5130) and $5 \mathrm{wt} \%$ carbon black (Timcal C45), and the graphite electrode is composed of $91.83 \mathrm{wt} \%$ graphite powder (Hitachi MagE3), 2 wt\% carbon black (Timcal C45), 6 wt\% PVDF binder (Kureha 9300) and $0.17 \mathrm{wt} \%$ oxalic acid. Single-crystal NMC is obtained from BTR China, and the nominal composition is $\mathrm{LiNi}_{0.84} \mathrm{Mn}_{0.06} \mathrm{Co}_{0.10} \mathrm{O}_{2}$. The single-crystal NMC electrodes were prepared by mixing NMC powder, PVDF binder and Super P Li carbon black in a mass ratio of 90:5:5 in a Thinky planetary mixer at $2000 \mathrm{rpm}$ for 10 minutes in total $(5$ minutes per cycle and two cycles). A 5 minutes sonication was added in between the two cycles to improve the dispersion and cool down the slurry. The cast electrode sheet was pre-dried in a dry-room at $100{ }^{\circ} \mathrm{C}$ for one hour to remove the NMP solvent.

$\mathrm{NMC}$ and graphite laminates were then punched into $14 \mathrm{~mm}$ and $15 \mathrm{~mm}$ circular disks, respectively, and dried further at $120^{\circ} \mathrm{C}$ for $12 \mathrm{~h}$ under dynamic vacuum $\left(\sim 10^{-2} \mathrm{mbar}\right)$ in Büchi oven. Dried electrodes were transferred to glovebox without air-exposure. The electrolyte in this work was $1 \mathrm{M} \mathrm{LiPF}_{6}$, EC/EMC 3/7 (LP57, SoulBrain MI, USA) with and without $2 \% \mathrm{VC}$, and the and separator was Celgard 3031. The Celgard separator was punched into $16 \mathrm{~mm}$ disks, washed in ethanol three times and dried at $50{ }^{\circ} \mathrm{C}$ under vacuum.

Battery assembly and cycling. Long-term cyclability were all performed in NMC/graphite full-cells using 2032 coin cells (Cambridge Energy Solution). Coin-cells were assembled in an Ar-atmosphere $\left(\mathrm{O}_{2}<1 \mathrm{ppm}, \mathrm{H}_{2} \mathrm{O}<1 \mathrm{ppm}\right)$ glovebox. Full cells were composed of a $14 \mathrm{~mm}$ NMC cathode, $16 \mathrm{~mm}$ separator and $15 \mathrm{~mm}$ graphite anode, and $50 \mathrm{~mL}$ electrolyte was added to each coin cell. The electrolyte used in polycrystalline NMC/graphite full-cells was LP57 (no $\mathrm{VC}$ ) and that in single-crystal NMC/graphite cells was LP57 with $2 \% \mathrm{VC}$. The cells were aged at a rate of $\mathrm{C} / 2$ (calculated based on a reversible capacity of $200 \mathrm{mAh}$ g-1 of NMC cathode) between $4.2 \mathrm{~V}$ and $2.5 \mathrm{~V}$ at room temperature $\left(20 \pm 1^{\circ} \mathrm{C}\right)$ using CCCV (constant current constant voltage) charging and $\mathrm{CC}$ (constant current) discharging protocol. $\mathrm{CCCV}$ charging was composed of a constant current step followed by a constant voltage hold at the upper cutoff voltage, i.e. $4.2 \mathrm{~V}$, until the current dropped below the value of $\mathrm{C} / 20$. Prior to the ageing cycles, one tap charge process and two formation cycles were applied. Tap charge was performed by holding the cells at $1.5 \mathrm{~V}$ for $15 \mathrm{~min}$, followed by a long rest of $6 \mathrm{~h}$ to allow cells to be fully wetted. ${ }^{27}$ Formation cycles were conducted at $\mathrm{C} / 20$ rate also using $\mathrm{CCCV}$ charging (current limit of the CV step is $\mathrm{C} / 40$ ) and $\mathrm{CC}$ discharging protocol.

For post-mortem samples at certain voltage or state-of-charge after extended ageing, fullcells were disassembled inside a glovebox, and the electrodes of interested were re-assembled into half-cells with Li metal as the counter electrode. The half-cells were then charged or discharged to the desired state.

Characterisation. Powder X-ray diffraction (PXRD): Laboratory PXRD was measured 
using a PANalytical Empyrean diffractometer $(\mathrm{Cu}$ Ka radiation, non-monochromatic, $\lambda=1.541$ $\AA$ ) in Bragg-Bento geometry. Samples were sealed in air-tight sample holder during the experiments. High-resolution synchrotron radiation (SR) PXRD measurements were conducted at beamline I11 at the Diamond Light Source using a wavelength of either $\approx 0.494$ or $\approx 0.8266 \AA$. Post-mortem power samples were scraped off from electrodes and packed into a $0.5 \mathrm{~mm}$ quartz capillary inside an Ar-glovebox. The capillaries were sealed using a twocomponent epoxy to prevent air-exposure.

The long-duration operando SR-PXRD was conducted on an NMC/graphite full-cell in modified coin-cell (Scitech Precision Ltd.) at beamline I11-1 (long-duration experiments LDEs facility) at the Diamond Light Source. ${ }^{28}$ For the modified coin-cell, the centre of the top and bottom casings are thinned down to $50 \mu \mathrm{m}$ in thickness using a laser beam to minimise beam attenuation. The LDE coin cell was mounted on a motorised stage in the LDE experimental hutch where the electrochemical ageing of the cell was conducted. The ageing was performed under the same protocol used for in-house coin cell cycling introduced above: two formation cycles at a rate of $\mathrm{C} / 20$ and long-term ageing at a rate of $\mathrm{C} / 2$. Before each of the dedicated beam time started, the sample stage was driven to the measurement position of the coin cell. Each pattern was collected with an exposure time of 5 minutes using a 2D Pixium area detector (X-ray energy $\approx 0.494 \AA$ ). Before each set of data acquisition, NIST Standard Reference Material $\mathrm{CeO}_{2}$ (NIST SRM 674b) was measured to obtain the X-ray wavelength and detector distance. Due to the irregularity of the available beam time, for instance, long shutdown due to synchrotron maintenance, the number of cycles during which diffraction patterns were collected were not controlled. In the present work, three datasets were recorded at cycle 348 , 439 and 915 , respectively.

Rietveld refinement of NMC cathode materials was performed in TOPAS (version 4.1) software, and based on the $\alpha-\mathrm{NaFeO}_{2}$ structural model $(R \overline{3} m$ space group, also known as the O3 structure using Delmas' annotation ${ }^{20}$ ) with the transition metals on the $3 \mathrm{a}$ sites, Li on the $3 \mathrm{~b}$ sites and $\mathrm{O}$ on the $6 \mathrm{c}$ sites. For NMC samples at discharged state, lattice parameters and oxygen position were refined with isotopic lattice strain while atomic displacement parameters (ADPs) are assumed to be isotropic and fixed at $1 \AA^{2}$ for all sites. For high SoC NMCs, both anisotropic lattice strain (Stephen's anisotropic peak broadening model), as well as anisotropic ADPs were included in the refinement. To estimate the Li content in electrochemically aged $\mathrm{NMC}$ cathode, the ratio of $c / a$ lattice parameters from the refinement and correlated with that on the first galvanostatic charge reported in our previous study. ${ }^{12}$ We note that the SoC cannot be correctly derived based on the $c / a$ or $c$ parameter alone as it evolves non-monotonically on charge or discharge, so the lattice parameter $a$ and the unit cell volume must also be considered in order to verify whether this $c / a$ parameter corresponds to the low or high SoCs.

Solid-state nuclear magnetic resonance (ssNMR): NMC cathodes for ssNMR experiments were electrochemically cycled in coin cells and extracted at the desired SoC. The cathode electrodes were rinsed with DMC three times $(\sim 0.5 \mathrm{~mL}$ each time $)$ and dried under vacuum for $30 \mathrm{~min}$. The cathode power was then scraped off from the Al current collector and packed into a $1.3 \mathrm{~mm}$ magic-angle spinning (MAS) NMR rotor in an Ar-glovebox. ${ }^{7} \mathrm{Li}$ ssNMR experiments were conducted on a $4.7 \mathrm{~T}\left(200 \mathrm{MHz}{ }^{1} \mathrm{H}\right.$ Larmor frequency) Bruker Avance III 
spectrometer, using a Bruker $1.3 \mathrm{~mm}$ double-resonance probe and under $60 \mathrm{kHz}$ magic-angle spinning. Recycle delays used were sufficiently long for quantitative analysis of the $\mathrm{Li}$ in NMC (typically 20-30 ms). ${ }^{7} \mathrm{Li}$ chemical shifts were referenced using $\mathrm{Li}_{2} \mathrm{CO}_{3}$ at $0 \mathrm{ppm}$. To obtain $7 \mathrm{Li}$ spectra without sidebands, the projection magic angle turning phase-adjusted spinning sidebands (pjMATPASS) sequence was used with eight $t_{1}$ increments. Variable temperature (VT) spectra were recorded using a rotor-synchronized Hahn echo pulse sequence with a total echo length of two rotor periods $(33.33 \mu \mathrm{s})$. Temperature calibration was based on chemical shift of ${ }^{207} \mathrm{~Pb}$ in $\mathrm{Pb}\left(\mathrm{NO}_{3}\right)_{2}{ }^{29}$

Electron microscopy: Scanning electron microscopy (SEM) experiments were performed on printed electrodes using a TESCAN MIRA3 FEG-SEM at $5.0 \mathrm{kV}$ with In-Beam secondary electron detection. Therefore, conductive carbon additives, which are $\sim 50 \mathrm{~nm}$ spherical particles, are present in the obtained images. The transmission electron microscopy (TEM) samples were prepared in the Ar glove box and placed on 300-mesh lacey-carbon-coated Au grids (Ted Pella). The dark field scanning transmission electron images (DF-STEM) were taken with an aberration-corrected JEOL JEM-2100 operated at 200kV. The high-angle annular darkfield imaging (HAADF) nominal probe size was $\sim 0.1 \mathrm{~nm}$. 


\section{Results and discussion}

\section{Phase segregation in polycrystalline NMC at high SoCs}

The crystal structure of the pristine NMC811 material is examined by high-resolution synchrotron-radiation X-ray diffraction, and the obtained diffraction pattern (presented in Figure 1a) shows an excellent fit to the structural model $(R \overline{3} \mathrm{~m}$, details of the refinement results are summarised in Table $\mathrm{S} 1$ ). $\mathrm{Li} / \mathrm{Ni}$ anti-site mixing, i.e. $\mathrm{Ni}$ on the $3 \mathrm{~b}$ sites in the Li layer (sometimes accompanied by $\mathrm{Li}$ in the metal layers), is a commonly observed phenomenon in Ni-rich layered materials due to similar size of $\mathrm{Li}^{+}$and $\mathrm{Ni}^{2+}$ but is minimal in the NMC811 used in this work $(\sim 1 \% \mathrm{Ni}$ in the Li layer), confirming the high quality of the sample. Figure 1(b) shows the typical morphology of NMC811 particles, which are $\mu \mathrm{m}$-sized spherical-shaped agglomerates composed of a large number of smaller primary particles. This is the most common morphology of industry-standard NMC materials synthesised via co-precipitation method and is referred to polycrystalline NMC in this work.
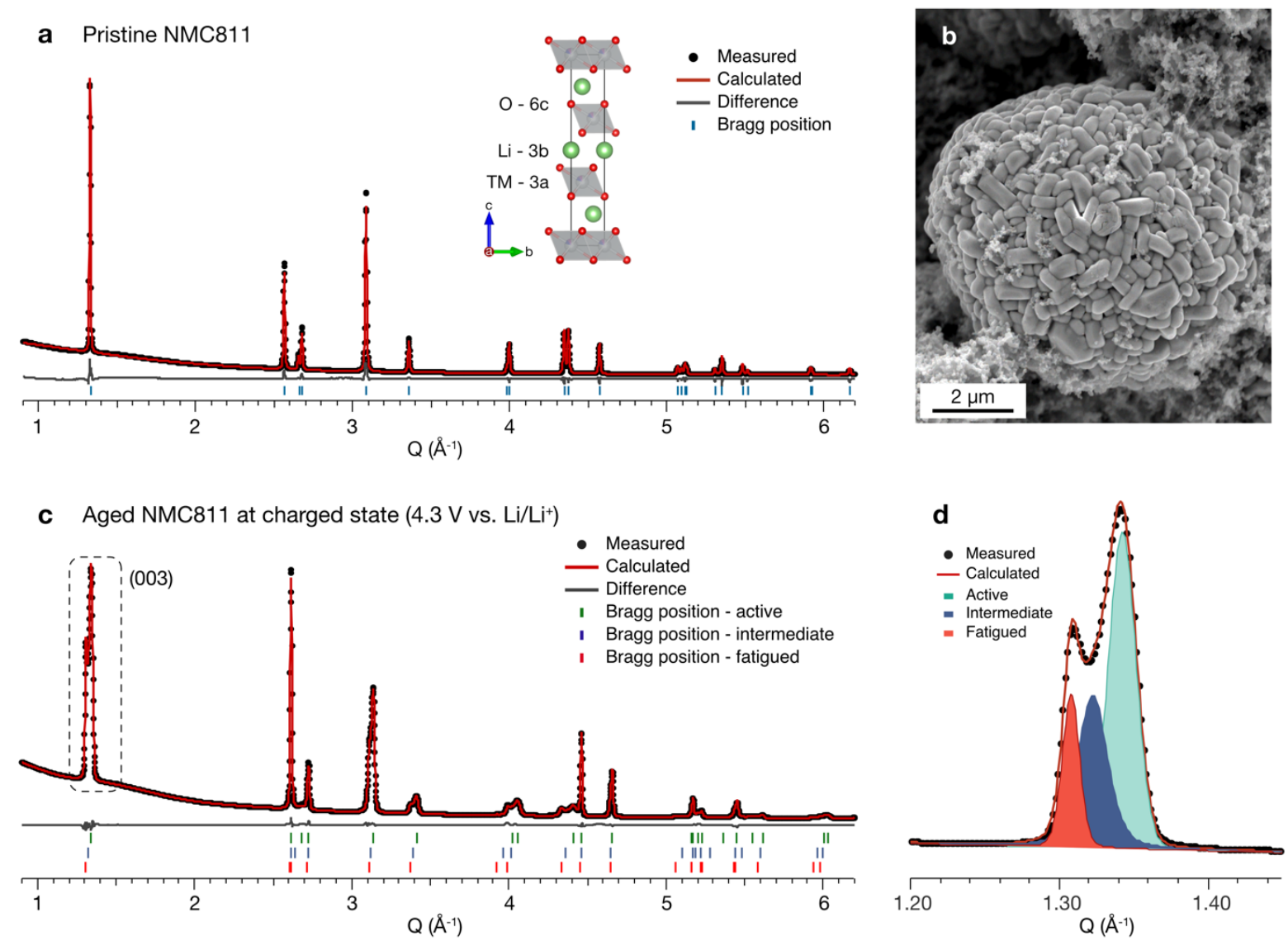

Figure 1. Pristine and electrochemically aged polycrystalline NMC811. a, Diffraction pattern and b, SEM image of a pristine polycrystalline NMC811 electrode. c, Diffraction pattern of an electrochemically aged NMC at a charged state $\left(4.3 \mathrm{~V} \mathrm{vs.} \mathrm{Li} / \mathrm{Li}^{+}\right)$after 300 cycles along with fit performed using three rhombohedral $R \overline{3} \mathrm{~m}$ phases with different lattice parameters (termed active, intermediate and fatigued). The final SoC was reached by charging the aged $\mathrm{NMC}$ in a half-cell at a rate of $\mathrm{C} / 20$ with a voltage hold until the current dropped below $\mathrm{C} / 1000$. d, Expansion of the region containing the (003) reflection.

To investigate any potential structural change in the bulk of the material induced by 
extended cycling, NMC811 cathodes were aged in full-cells using graphite as the anode, and their typical cycling performance is summarised in Figure S1. Briefly, the first ageing cycle (excluding two formation cycles) shows a discharge capacity of $181 \mathrm{mAh} / \mathrm{g}$ at $\mathrm{C} / 2$ rate and gradually decreases to $110 \mathrm{mAh} / \mathrm{g}$ after 1000 cycles, representing capacity retention of $60.8 \%$, where the capacity is calculated based on the mass of the cathode. A continuous increase in the voltage polarisation between charge and discharge is observed as demonstrated in Figure S1b, which shows the voltage profile at various ageing cycles. This is associated with the cell impedance growth which is a commonly-observed phenomenon in Ni-rich NMC cells. ${ }^{9,30}$

The bulk structure of electrochemically aged NMC is examined at both discharged (i.e. lithiated) and charged (i.e. delithiated) states. To reach the final desired SoC, aged NMC materials were re-assembled into a half-cell with $\mathrm{Li}$ metal as the anode followed by electrochemically charged/discharge. In the discharged state, NMC shows no sign of bulk structural transformation after 300 ageing cycles and the powder diffraction pattern can be fit using Rietveld refinement with only one rhombohedral $R \overline{3} m$ phase (Figure $\mathrm{S} 2$ ). This is in good agreement with previous literature reports that have shown that layered NMC and NCA cathodes remain single-phase at the discharged state even after long-term ageing. ${ }^{9,24}$

However, NMC at a charged state exhibits a more complex diffraction pattern as shown in Figure 1c. In contrast to the single-phase characteristics of NMC811 during the initial cycles, ${ }^{11,12,31}$ evident peak splitting is observed for multiple reflections, for instance, the (003) reflection (Figure 1d) indicating the presence of multiple crystallographic phases. To distinguish and index these various phases, Rietveld refinement was carried out with three rhombohedral R3-m phases under the assumption that the symmetry remains unchanged. More details describing the basis for this assumption are given in the Supporting Information. The number of rhombohedral phases included in the refinement, i.e. three in the present case, is estimated based on the line-shape of the (003) reflection and represents the simplest fit, with the least number of variables, to the data. The fit to the experimental data is excellent (the refinement result is summarised in Table S2), confirming that the multicomponent structure can be described via a model containing rhombohedral, O3, NMC phases only, without needing to include a component from another phase (e.g., an $\mathrm{O} 1$ phase); the $\mathrm{O} 3$ phases are associated with clearly discernible difference in lattice parameters, which can be ascribed to variations in the state of charge, or in other words, the different residual Li content in the structure. ${ }^{12}$

The $\mathrm{Li}$ content ( $\mathrm{y}$ in $\mathrm{Li}_{\mathrm{y}} \mathrm{Ni}_{0.8} \mathrm{Mnn}_{0.1} \mathrm{C}_{0.1} \mathrm{O}_{2}$ ) is estimated based on the ratio of $c / a$ lattice parameters obtained from the refinement (further details of the analysis are described in the Experimental), and the corresponding values of the three $\mathrm{O} 3$ phases, from $\mathrm{Li}$ rich to $\mathrm{Li}$ poor, are $25.5 \%, 20.1 \%$ and $15.0 \%$, respectively. As the NMC at charged state represents a delithiated state, a higher Li-content indicates a sluggish delithiation. In this work, the NMC phase which shows the lowest degree of delithiation (i.e. lowest SoC) is described as being "fatigued" whereas the ones which have intermediate and highest SoC are referred as "intermediate" and "active”. For the delithiated NMC at high SoCs ( $65 \%$ and higher), the (003) peak at lowest Q (i.e. the scattering factor length) is attributed to a least delithiated O3 phase i.e, the fatigued NMC phase. 


\section{Structural evolution of polycrystalline NMC811 during cycling}

To understand the formation and evolution of the fatigue process, operando long-duration synchrotron PXRD was performed on the polycrystalline NMC811/graphite in a fully sealed coin cell at regular intervals throughout the long-term ageing (Figure 2a). The operando coin cell with the laser-thinned X-ray window achieved more than 1000 cycles at a cycling rate of $\mathrm{C} / 2$ (Figure $2 \mathrm{~b}$ ) confirming that this novel cell design is an excellent tool for studying structural evolution over long-term cycling. The NMC811/graphite full-cell exhibited a discharge capacity of $184.6 \mathrm{mAh} / \mathrm{g}$ at the first $\mathrm{C} / 2$ cycle after the two formation cycles and $117.8 \mathrm{mAh} / \mathrm{g}$ at the $1000^{\text {th }}$ cycle, representing capacity retention of $63.8 \%$.
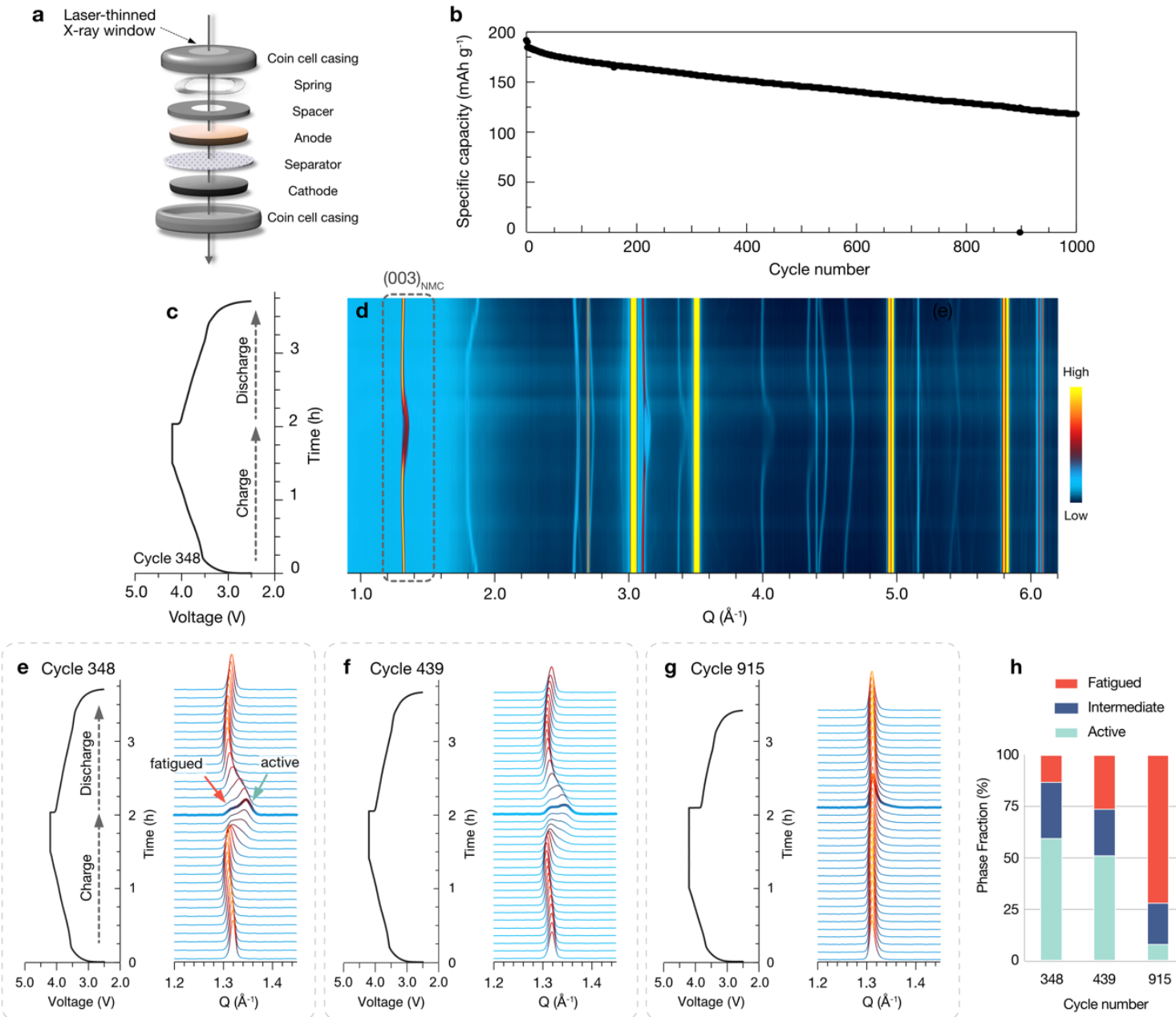

Figure 2. Operando long-duration PXRD investigation of an NMC811/graphite full-cell. a, A schematic drawing of the laser-thinned operando coin cell. b, Capacity retention performance of the operando coin cell. c, Voltage profile during cycle 348 and d, the corresponding diffraction patterns. Evolution of the NMC (003) reflection at cycle 348 (e), cycle 439 (f) and cycle 915 (g). The thickened line is the diffraction pattern collected at the end of the charging step. $\mathbf{h}$, Quantification of the fatigued, intermediate and active NMC phases at cycle 348, 439 and 915. 
Figure 2c shows the voltage profile of the NMC811/graphite cell at cycle 348, which comprises a constant current (CC) charge from $2.5 \mathrm{~V}$ to $4.2 \mathrm{~V}$, followed by a constant voltage $(\mathrm{CV})$ hold at $4.2 \mathrm{~V}$, and then $\mathrm{CC}$ discharge to $2.5 \mathrm{~V}$. The corresponding diffraction patterns are summarised in Figure $2 \mathrm{~d}$. Although the coin cell casings have been thinned down to $50 \mu \mathrm{m}$, the $\mathrm{X}$-ray beam is still strongly attenuated by the stainless steel, which itself gives rise to the strong signals at $\sim 3.03,3.50,4.95$ and $5.81 \AA^{-1}$. However, clear signals of the active materials, namely NMC811 and graphite, are observed, for instance, the (003) reflection of the NMC811 at $\sim 1.32$ $\AA^{-1}$ and the graphite/lithiated graphite between $\sim 1.8$ and $1.9 \AA^{-1}$. Detailed assignments of reflections are given in Figure S3. Moreover, the evolution of the reflection peaks is also clearly observed, confirming this coin cell is able to provide high-quality operando diffraction results as well as excellent long-term cyclability.

The ex-situ results in Figure 1 illustrate that the (003) reflection is an excellent proxy for identifying the phase segregation in NMC at high SoCs. The (003) peak position gradually shifts to lower Q during the first hour of charging (Figure 2e), i.e., from lower to intermediate SoCs, and then shifts back to higher $\mathrm{Q}$ at high SoCs. This is in good agreement with the results of Ni-rich NMCs in initial cycles reported in the literature. ${ }^{9,12}$ The substantial peak broadening at high SoCs, on the other hand, is a strong indication of the presence of multiple phases. The overall line shape is analogous to that of the high-resolution ex-situ pattern shown in Figure 1c and $\mathrm{d}$, which we successfully indexed with three rhombohedral $R \overline{3} m$ phases - again the smallest number of phases required to fit the pattern. Refinement against the pattern recorded at the end of charge in cycle 348 (thickened line in Figure 2e) confirms that all NMC particles remain in the $\mathrm{O} 3$ structure, and the $\sim 1.32 \AA^{-1}$ shoulder is associated with the NMC components that are higher in Li content, i.e. the fatigued phase. Moreover, the emergence of phase segregation occurs predominately during the CV step on charge.

Upon further ageing to the $439^{\text {th }}$ cycle, the fraction of the fatigued phase at the end of charge increased noticeably as evidenced by the increase in the relative intensity of its (003) peak as shown in Figure $2 \mathrm{f}$. By the $915^{\text {th }}$ cycle, the majority of the NMC material is significantly degraded and very little of the active phase observed. Evolutions of the lattice parameters of NMC during cycle 348, 439 and 915 obtained from Rietveld refinements are summarised in Figure S4. The position of the (003) peak did not change significantly in cycle 915. This reflects the fact the NMC cathode is only cycled over a limited range of SoC, approximately from $50 \%$ to $75 \%$ SoC for the fatigued phase, and that these changes of SOC are associated with very small differences in the $c$ lattice parameter (shown in Figure S4j). This is a result of the electrode potential slippage during the electrochemical ageing of full cells due to loss of lithium to the graphite. ${ }^{32}$

Figure $2 \mathrm{~h}$ summarises the phase fraction obtained from the Rietveld refinement against the results at the end of charge of all these three cycles. Although the errors in the phase fractions are quite large (a few percent) due to a large number of variables in the refinement and the 
poorer quality of the patterns compared the ex-situ data, the general trend agrees well with the change in the line shape: the fatigue phase increases and active phase decreases as the cycle number increases. It should also be noted that the fatigued phase is not entirely inactive during cycling as indicated by the continuing evolution of the (113) reflection of the NMC cathode shown in Figure S5 (further details of this analysis in Supporting Information Figure S5). It should also be noted the final phase fraction of the fatigued in the full-cells may vary with charge rates as this may result in different degrees of delithiation of the NMC and lithiation of the graphite at the end of charge. For instance, at fast rates, NMC cathodes typically show lower capacities as a result of incomplete delithiation, and this will lead to a higher amount of "apparent" fatigued phase in the diffraction results. This is particularly true for the late cycles which have a higher cell impedance. Indeed, when the NMC cathode from this LDE coin cell was extracted after 1200 cycles, and then charged at extremely slow condition after being assembled into a fresh half-cell, the ratio of the fatigued and the active phase is now approximately 50:50 (Figure S6; charging condition: $\mathrm{C} / 20$ with a voltage hold at $4.3 \mathrm{~V}$ vs. Li until the current is lower than $\mathrm{C} / 1000$ ). However, the qualitative trend of an increase in the fraction of the fatigued phase obtained from the LDE cell is still clear and valid. Therefore, our results suggest that repetitive cycling leads to the growth of the fatigued phase, which is still electrochemically active but only to a limited SoC.

\section{Fatigued phase as a function of cycle number: high similarity in lattice parameters}

Table 1. Summary of lattice parameter c/a ratios and Li contents of the fatigued phase as a function of cycle number obtained for the polycrystalline sample in different experiments.

\begin{tabular}{lcccc}
\hline & $\begin{array}{c}\text { Cycle } \\
\text { number }\end{array}$ & $c / a$ & $\begin{array}{c}\mathrm{Li} \text { content } \\
(\mathrm{y} \text { in } \\
\left.\mathrm{Li}_{\mathrm{y}} \mathrm{Ni}_{0.8} \mathrm{Mn}_{0.1} \mathrm{Co}_{0.1} \mathrm{O}_{2}\right)\end{array}$ & $\begin{array}{c}\text { State-of-charge } \\
\left(\mathrm{x} \mathrm{in} \mathrm{Li}_{(1-}\right. \\
\left.{ }_{x} \mathrm{Ni}_{0.8} \mathrm{Mn}_{0.1} \mathrm{Co}_{0.1} \mathrm{O}_{2}\right)\end{array}$ \\
\hline Synchrotron: ex-situ & 300 & $5.1114(7)$ & $25.5(1) \%$ & $74.5(1) \%$ \\
\hline $\begin{array}{l}\text { Synchrotron: } \\
\begin{array}{l}\text { operando long- } \\
\text { duration experiment }\end{array}\end{array}$ & 348 & $5.110(7)$ & $25(1) \%$ & $75(1) \%$ \\
\hline $\begin{array}{l}\text { Lab diffractometer: } \\
\text { ex-situ }\end{array}$ & 615 & $5.110(4)$ & $25.3(6) \%$ & $74.7(6) \%$ \\
\hline
\end{tabular}

Interestingly, the maximum accessible $\mathrm{SoC}$ of the fatigued phase observed in the operando long-duration PXRD experiment does not change significantly from cycle 348 to 439 and 915 , as evidenced by the peak position of the $(003)$ reflection of the fatigued phase in these three cycles at the end of charge: $\sim 1.33 \AA^{-1}$ in cycle $348, \sim 1.33 \AA^{-1}$ in cycle 439 and $\sim 1.31 \AA^{-1}$ in cycle 915 , respectively (Figure $2 \mathrm{e}, \mathrm{f}, \mathrm{g}$ ). This is a strong indication that the $\mathrm{SoC}$ of these three fatigued phases is very close. Indeed, the deviation of the $c / a$ ratios of the fatigued phases in 
six samples (listed in Table 1) is extremely small, which suggests that the unit cell parameters as well as the residue Li contents of these fatigued phases are very similar. There is a minor discrepancy of only $\sim 0.5 \%$ in the estimated Li content and therefore the state of charge: all the fatigued phases are found at $\sim 75 \% \mathrm{SoC}$. The nearly identical lattice parameters and Li content for the fatigued phases at their maximum accessible $\mathrm{SoC}$ is a strong indication that there is a structural origin behind their electrochemical limitation.

\section{Long-range structure, Li local environments and mobility}

Antisite mixing: The diffraction results discussed above confirm that, in the bulk, no phase transformation occurs, and the materials remain layered. In this section, we evaluate more subtle changes to the bulk crystal structure. Makimura et al. systematically studied the effect of the degree of $\mathrm{Li} / \mathrm{Ni}$ anti-site mixing on the electrochemical performance of NCA cathodes and convincingly showed that the presence of $\mathrm{Ni}$ in the Li layer impedes Li-ion transport and limits the reversible capacity. ${ }^{33}$ To assess the role of $\mathrm{Li} / \mathrm{Ni}$ antisite mixing in the formation of the fatigued phase Rietveld refinements were performed on aged NMC811 samples in the discharged state (i.e., lithiated) and antisite mixing results are summarised in Figure 3a. The value of the pristine NMC here shows a small deviation from that of synchrotron measurement as listed in Table S1; this is most likely due to the poorer quality of the laboratory data. Nevertheless, by comparing the measurements from the same instrument, a qualitative analysis of whether the anti-site mixing increases or not is valid. A minor variation of the percentage is observed among the pristine and aged cathodes. The calculated value shows a minor variation from the pristine state to 1200 ageing cycles, and this is in good agreement with prior results of Friedrich et al. that no increase in the Li/Ni anti-site mixing is observed in NMC811 after $\sim 1000$ electrochemical ageing cycles. ${ }^{9}$ 

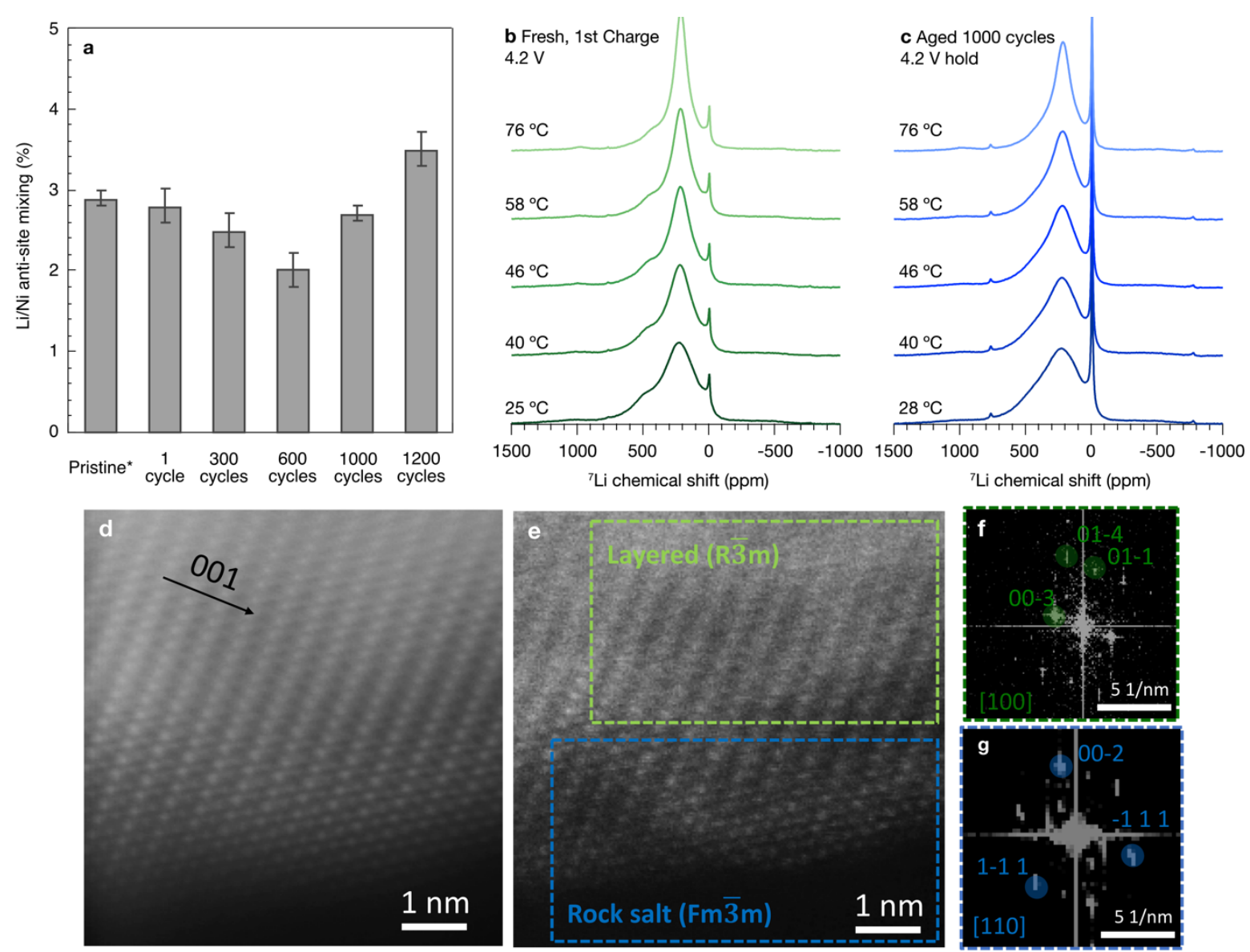

Figure 3. Structural analysis of fresh and aged NMC811 cathodes. a, Summary of the Li/Ni anti-site mixing as a function of the cycle number obtained from Rietveld refinement against laboratory diffraction data. b,c, ${ }^{7} \mathrm{Li}$ ssNMR spectra of fresh (b) and aged (c) NMC cathodes acquired at variable temperatures at $4.7 \mathrm{~T}$ and $60 \mathrm{kHz}$ MAS frequency. d,e, DF-STEM images of aged NMC811 particle after FFT filtering (d) and the original DF image before FFT filtering (e). f, FFT of the upper and the bottom regimes indicate the crystal orientation of the layered rhombohedral $R \overline{3} m$ structure in along the $<100>$ zone axis and (g) face-centered cubic structure along the $<110>$ zone axis, respectively. The NMC sample used for STEM imaging was aged for 300 cycles, and the final SoC was at $4.3 \mathrm{~V}$ vs. Li.

$N M R:{ }^{7} \mathrm{Li}$ ssNMR is a powerful tool to investigate the Li local environment in Li-ion battery materials as well as provide information on the Li-ion dynamics. ${ }^{12,34}$ We recently reported that the Li-ion mobility in NMC811 varies noticeably depending on the SoC: it gradually increases from the fully lithiated state to $\sim 40 \% \mathrm{Li}$ removal and remains at a high level until $65 \%$; this is followed by a decrease on further delithiation. ${ }^{12}$ Therefore, in order to probe changes of Liion mobility after cycling, we examined Li mobility in fresh and aged samples at the maximum achievable SoC for the fatigued NMC ( $75 \%$ Li removal, $4.2 \mathrm{~V}$ vs. Li). We chose this SoC for two reasons: (1) if sluggish Li motion is the limiting factor which prevents the fatigued phase from further delithiation, a major decrease in Li mobility should be observed in the aged samples; (2) the long-range structure remains single phase at this SoC, facilitating the 
interpretation of the results.

Figure $3 \mathrm{~b}$ and $\mathrm{c}$ show the ex-situ ${ }^{7} \mathrm{Li}$ solid-state NMR spectra as a function of temperature of a fresh NMC at $4.2 \mathrm{~V} \mathrm{(b)}$ and an aged NMC at $4.2 \mathrm{~V}$ after 1000 cycles (c). Both samples are indeed single phase as confirmed by XRD and diffraction patterns are shown in Figure S7a and b. Structural refinement also confirms that the two samples have similar lattice parameters and therefore SoC. In all the ${ }^{7} \mathrm{Li}$ NMR spectra, the sharp signal at $\sim 0 \mathrm{ppm}$ is attributed to diamagnetic $\mathrm{Li}$ salts, for instance, residual $\mathrm{LiPF}_{6}$ and Li-containing cathode-electrolyteinterphase (CEI) compounds such as $\mathrm{Li}_{\mathrm{x}} \mathrm{PF}_{\mathrm{y}} \mathrm{O}_{\mathrm{z}}$ and $\mathrm{Li}_{2} \mathrm{CO}_{3}$, which are formed as a result of electrolyte decomposition during cycling. ${ }^{18,19}$

The broader signals at higher chemical shifts originate from the Li in the NMC cathode material. The observed shift is predominately attributed to the Fermi-contact interaction between $\mathrm{Li}$ and the unpaired electrons on the paramagnetic transition metal ions through the bridging oxygen ions, and the broad line-shape is a result of a large number of paramagnetic species and wide distribution of different Li local environments. ${ }^{12,34,35}$ For both the fresh and the aged $\mathrm{NMC}$ at $4.2 \mathrm{~V}$ (Figure $3 \mathrm{~b}$ and $\mathrm{c}$ ), the integral of the signal from paramagnetic $\mathrm{Li}$ is similar, confirming that the two samples are close in SoC. After extended cycling, the lineshape is qualitatively similar to the fresh sample, although the distinct shoulder at around $500 \mathrm{ppm}$, which has been assigned to $\mathrm{Li}$ in sites with two $\mathrm{Mn}^{4+}$ ions in the first coordination shell, ${ }^{12}$ is no longer as well resolved in the aged sample (Figure $3 \mathrm{~b}$ and $\mathrm{c}$ ). The broadening of this feature can be attributed to slightly different states of charge across the sample, with different particles having slightly different lattice parameters and oxidation states, as seen previously for NCA. ${ }^{36}$

As outlined in our previous work, Li motion strongly affects the observed NMR spectra since fast hopping between different Li sites results in sharpening of the resonances. ${ }^{12}$ In our work on NMC811, this effect can be observed for hopping frequencies greater than $\sim 20 \mathrm{kHz} .{ }^{12}$ With a temperature increase from below $30{ }^{\circ} \mathrm{C}$ to $76{ }^{\circ} \mathrm{C}$, the ${ }^{7} \mathrm{Li}$ spectra of both the fresh and aged sample show a noticeable narrowing in line-width, indicating faster Li motion in both samples at higher temperatures. To compare Li-ion mobility in the two samples, we modelled the experimental spectra based on our approach developed previously (Figure S8). ${ }^{12}$ An outline of this approach and the approximations used are given in the SI. In brief, the experimental spectra are approximated as the sum of two spectra, being caused by Li ions with low and high mobility, respectively. High-mobility ions are defined here as having a hopping frequency of more than $20 \mathrm{kHz}$, leading to spectral line narrowing as described above. The model confirms that $\mathrm{Li}$ hopping rates increase with temperature for the high mobility ions (Figure S9a,b). Their overall hopping rate appears to be lower in the aged sample, an observation which is supported by slightly longer transverse relaxation times in this sample (Table S3). Nevertheless, our model suggests that the ratio of low- and high-mobility Li ions is very similar in both samples, roughly 50:50, and that the fraction of high-mobility ions in the aged sample is only slightly smaller than in the fresh sample, with a difference not greater than $\sim 10 \%$ (Figure S9c). Although we were unable to quantify the phase fraction of this particular sample because the fatigued and the active phases have the same lattice parameters at this SoC, another long-term 
aged sample (1200 cycles, Figure S6) shows a ratio of approximately 50:50, and thus it is reasonable to assume that the aged sample discussed here (1000 cycles) also contains a significant proportion of the fatigued phase. Therefore, the minor difference in the faction of high-mobility Li ions suggests that the fatigued phase is not characterized by Li-ions with drastically lower mobility. To conclude, while the overall mobility is reduced in the aged sample, a similar proportion of Li-ions still appears to be in the high mobility regime when compared to the fresh sample. This indicates that it is unlikely that a lack of sufficiently mobile $\mathrm{Li}$ ions in the aged sample causes the fatigue degradation.

In addition to the two $4.2 \mathrm{~V}$ samples discussed above, ${ }^{7} \mathrm{Li}$ NMR experiments were also carried out on an aged NMC sample (1200 cycles) that was charged to $4.3 \mathrm{~V}$ vs. Li to investigate the distribution of Li local environments. As expected, the sample shows a coexistence of the fatigued and active phases as shown by XRD (Figure S6). Moreover, a sharpening of the signal is observed in the variable temperature NMR spectra upon heating, suggesting that mobile ions remain in the fatigued NMC sample (Figure S11). The results indicate that that the Li-ions in the bulk of electrochemically aged NMC are still mobile and decreasing Li-ion mobility of the bulk phase is not the major cause of the fatigue degradation.

TEM analysis: Although no phase change or increase in antisite mixing in the bulk of the material is observed, an evident structure transformation on the surface of electrochemically aged NMC is identified as shown in the DF-STEM images in Figure 3d and e obtained for a sample after 300 ageing cycles with the subsequent charging to a final SoC state of $4.3 \mathrm{~V}$ vs. Li. The top section (green dashed box) of the STEM images is attributed to the layered NMC (oriented along the $<100>$ zone axis) as confirmed by the Fast-Fourier-Transformation (FFT) analysis presented in Figure $3 \mathrm{f}$ whereas the bottom section (blue box) is indexed as a rock salt, NiO-like phase. The surface rock salt layers have grown close-to-epitaxially on the edges of the layered phase particles. A more detailed analysis of other regions and samples reveals rock salt phases covering the primary particles (Figure S12). For instance, a surface layer is shown growing parallel to the (003) lattice fringes (i.e., layers) as presented in Figure S13. These findings in a good agreement with literature: the surface of layered cathodes tends to undergo a structural transformation from layered to spinel and rock salt after electrochemically cycling and even following contact with electrolyte. ${ }^{13,14,16,37}$

\section{Phase segregation in single-crystal NMC at high SoCs}

Two often cited explanations in the literature for the phase segregation behaviour observed in layered Li transition metal oxide cathodes are: (1) inter-granular cracking, ${ }^{26,30}$ (2) non-uniform impedance growth after extended electrochemical ageing ${ }^{30}$ due to the formation of resistive surface reconstruction layer and/or cathode electrolyte interphase (CEI). In this work, we employed single-crystal NMC materials which should be much less prone to inter-granular cracking, and performed delithiation on electrochemically aged cathodes at an extremely slow 
rate to test these two hypotheses.

The single-crystal NMC samples show the typical morphology of the single-crystal NMC, which comprise several agglomerated $\mu \mathrm{m}$-sized large particles, with many fewer grain boundaries than the polycrystalline sample (Figure 4a). High-resolution PXRD confirms that the single-crystal NMC is phase pure and with $\sim 2.4 \% \mathrm{Ni}-\mathrm{Li}$ anti-site mixing (Figure S14a; Rietveld refinement results are summarised in Table S4). Electrochemical ageing of the singlecrystal NMC was conducted under the same conditions as the polycrystalline NMC811, and typical capacity retention performance and voltage profiles are summarised in Figure S14. The discharge capacity at a $\mathrm{C} / 2$ rate decreased from $170.0 \mathrm{mAh} / \mathrm{g}$ to $116.5 \mathrm{mAh} / \mathrm{g}$ after 500 cycles.
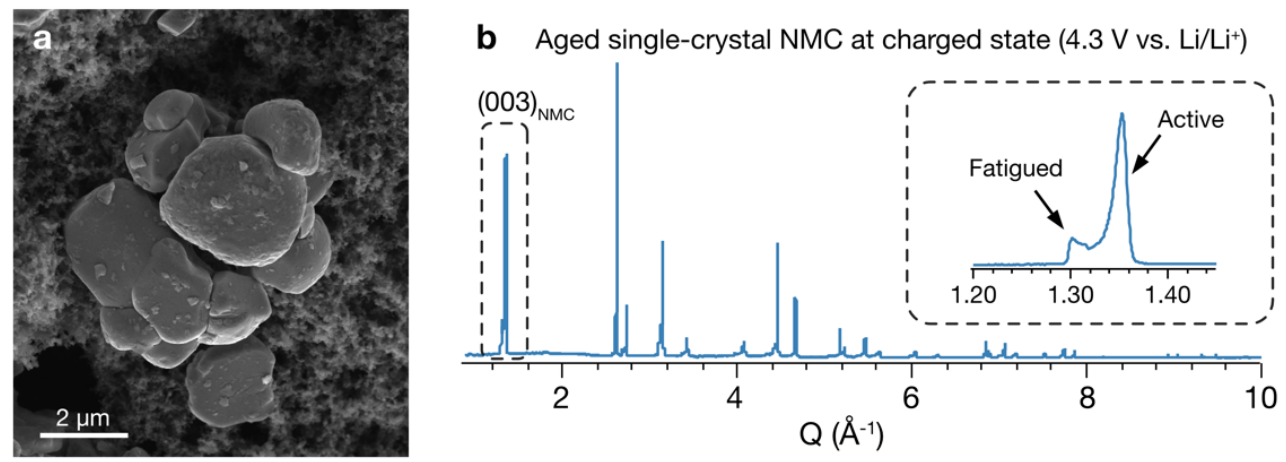

Figure 4. Single-crystal NMC cathodes. a, SEM image of the single-crystal NMC electrode. b, Diffraction pattern of electrochemically aged single-crystal NMC after 500 cycles at charged state $\left(4.3 \mathrm{~V}\right.$ vs. $\left.\mathrm{Li} / \mathrm{Li}^{+}\right)$. The final $\mathrm{SoC}$ was reached by charging the aged $\mathrm{NMC}$ in a half-cell at a rate of $\mathrm{C} / 100$ with a voltage hold until the current dropped below $\mathrm{C} / 1000$. Inset: expanded (003) reflection region to illustrate peak splitting.

The aged single-crystal NMC was charged to high SoC (4.3 V vs. Li), and its diffraction pattern is shown in Figure 4b. Similar to the polycrystalline NMC811 electrode, single-crystal NMC also shows an obvious phase segregation as evidenced by the splitting of the (003) peak (inset in Figure $4 \mathrm{~b}$ ), even though the final delithiation was carried out at an extremely slow rate of $\mathrm{C} / 100$ with constant voltage hold. The strong peak at $\sim 1.353 \AA^{-1}$ is attributed to the active NMC phase which is at higher SoC than the rest. On the other hand, the signals at lower $\mathrm{Q}$ are associated with less reactive population, for instance, the signal at the lowest $\mathrm{Q}$ of $\sim 1.301 \AA^{-1}$ is attributed to the NMC particles at lowest SoC and therefore are the most fatigued. It is also clear that multiple fatigued phases with distinct $c$ lattice constants exist in this aged material as evidenced by the broad distribution in $\mathrm{Q}$ values between the most fatigued and active NMCs. Due to the co-existence of a large of number of phases, attempts of Rietveld refinements were not successful so the Li-contents in them are not readily quantified. A qualitive estimate of the lattice parameters for the fatigued phase was made using the peak positions of certain reflections since as the zero point is negligible $\left(-0.000376^{\circ}\right.$ obtained from the beamline instrument calibration). Values of $c=14.48 \AA\left((003)\right.$ peak at $\left.1.301 \AA^{-1}\right)$ and $a=2.831 \AA((110)$ peak at $4.436 \AA^{-1}$ ) were extracted, corresponding to a $c / a$ ratio of 5.115 and a SoC of $\sim 74 \%$. These values are almost identical to those obtained for polycrystalline NMC 811. Moreover, 
this aged single-crystal NMC was charged to even higher SoC (4.6 V at a rate of C/100 rate and a constant voltage hold) and the diffraction pattern (presented in Figure S15b) shows that the fatigued NMC is still preserved at this high potential, the (003) peak position of the fatigued NMC phase remaining unchanged. The fact that the phase segregation is still observed in the aged single-crystal NMC charged at extremely slow rates strongly indicates that structural fatigue cannot be solely explained by inter-granular cracking or impedance growth of the smaller secondary particles.

\section{Discussion: Origin of the fatigued NMC}

The fatigued material is a layered phase that is still electrochemically active but only to a maximum SoC of $\sim 75 \%$. While mobile Li-ions remain in this phase (NMR), they cannot be extracted even under extremely slow delithiation and voltage hold at $4.6 \mathrm{~V}$ vs. Li. The surface reconstruction layer identified in the STEM images (Figure 3) is known to be poor Li-ion conductor and therefore can impede Li-ion transport. ${ }^{13,14,16,37}$ This negative effect is expected to be independent of the SoC as the electrochemically inactive rock salt oxides surface layers are not likely to change significantly during one cycle. Instead, this rock salt formation is responsible for the overall increase in impedance seen on cycling, which manifests itself in the increasing overpotential seen between charge and discharge. The phase segregation is a different phenomenon which is only observed at high SoCs (above $75 \%$ ) and therefore cannot be explained fully by the slow Li transport through the surface reconstruction layer.

The fact that the crystal structure of the surface rock salt remains unchanged due to its electrochemically inactive nature, while significant lattice expansion and contraction occurs to the layered structure in the bulk of the particles during cycling will, however, generate substantial dynamic lattice mismatch which we now discuss. This interfacial lattice strain will have a strong dependence on the SoC of the NMC cathode. A s the surface reconstruction layer can form on various NMC particle facets, the degree of mismatch will also depend on the relative orientation of the two structures. We consider the effect of scenario in which the surface reconstruction layer is formed orthogonal to the Li diffusion planes as illustrated in Figure 5a because: (1) more thicker surface reconstruction layers are generally seen for this orientation as compared to other orientations, ${ }^{14}(2)$ more importantly, the effect of the lattice mismatch is expected to higher than for other orientations, as the evolution of $c$ lattice parameter of the layered NMC811 is much larger than that of $a$ and $b$. The lattice mismatches were calculated using the following equations:

Lattice mismatch in the $c$ direction, $\varepsilon_{c},=\left(d_{R(003)}-d_{F(111)} * 6\right) * 100 \%$

Lattice mismatch in the $a$ direction, $\varepsilon_{a}=\left(d_{R(100)}-d_{F(111)}\right) * 100 \%$

where $d_{\mathrm{R}(003)}$ and $d_{\mathrm{R}(100)}$ are the d-spacings of the (003) and (100) lattice planes, respectively and $d_{\mathrm{F}(111)}$ is the d-spacing of the (111) plane of the cubic (Face-centered, F) NiO. The NMC811 lattice parameter values used here are from our previous study. ${ }^{12}$ The evolutions of $\varepsilon_{c}$ and $\varepsilon_{a}$ 
(Figure 5c and d) track the lattice constants a and c, because the $F(111) d$-spacing is constant throughout the cycling of NMC. Specifically, $\left|\varepsilon_{c}\right|$ gradually decreases from the beginning of charge to $\sim 65 \%$ SoC, where it reaches a minimum of nearly $0 \%$, and then increases on further delithiation to $\sim 3.5 \%$. By contrast, in the $a$ direction, $\left|\varepsilon_{a}\right|$ increases monotonically during the delithiation process remaining essentially constant above a SoC of $75 \%$. The overall interfacial lattice strain, therefore, becomes noticeably higher when bulk NMC reaches SoCs higher than $65 \%$ as both $\left|\varepsilon_{c}\right|$ and $\left|\varepsilon_{a}\right|$ are increasing. In particular, $\sim 75 \% \mathrm{SoC}$ is a distinct threshold above which the overall lattice mismatch becomes significantly higher as the rise in $\left|\varepsilon_{c}\right|$ accelerates sharply; $\left|\varepsilon_{a}\right|$ also increases from this point, although its change is less pronounced. It is also worth noting that such lattice mismatch becomes less important for certain orientations, for instance, rock salt formed parallel to the lithium layers (as shown in the TEM image in Figure S13) because $\left|\varepsilon_{c}\right|$ has no impact on this interface.

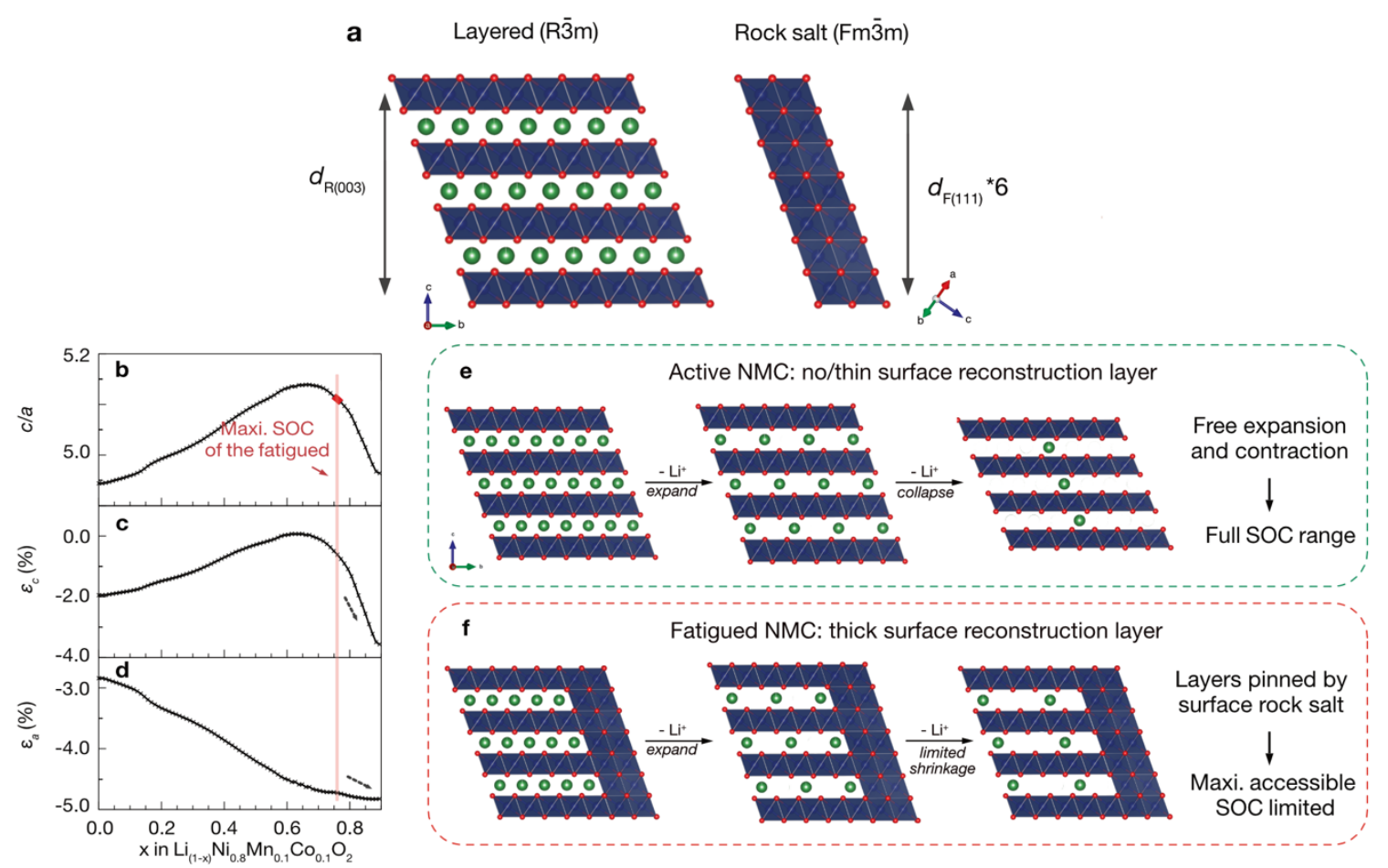

Figure 5. Interfacial lattice strain induced fatigue degradation of Ni-rich layered cathodes. a, Illustration of the layered structure along the $\langle 100\rangle$ zone axis and rock salt structure along the $\left\langle 110>\right.$ zone axis. b,c,d, c/a ratio (b), lattice mismatch in the $c$ direction $\varepsilon_{c}$ (c) and lattice mismatch in the $a$ direction $\varepsilon_{a}$ (d) as a function of state-of-charge. e,f, Illustration of the structural evolution of the (e) active phase, and (f) fatigued phase during delithiation.

Here, we propose that the fatigue degradation is due to the high lattice strain at the interface between surface and bulk when the NMC cathode at SoCs above the threshold of $75 \%$. Figure 5e illustrates structural evolution for the NMC with a thin or no rock salt surface layer, showing that the NMC lattice can expand and contract freely; therefore, the active material can achieve the full SoC range. In contrast, as illustrated in Figure 5f, when a thick rock salt layer is 
presented, the lattice planes of the layered structure at the interface are pinned by the rock salt due to high interfacial lattice strain, particularly in the $c$ direction. This prevents the layers from further contracting, which is however required for further delithiation. Macroscopically this is manifested as a fatigue phenomenon in which part of the cathode material becomes electrochemically active in a limited SoC window, i.e. between low SoCs and $\sim 75 \%$ SoC.

Moreover, the fact that such a fatigued phase can still be re-lithiated is because: (1) lithiation from $\sim 75 \%$ to a lower SoC (as illustrated in Figure 5 ) of close to $50 \%$ represents a decrease in the interfacial lattice mismatch, (2) the driving force for lithiation, is greater than that for delithiation, as evidenced by a more steep change in the potential during lithiation from $\sim 75 \%$ to a lower SoC, as opposed to delithiation above $75 \%$ SOC.

Pristine NMC811
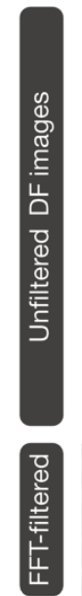
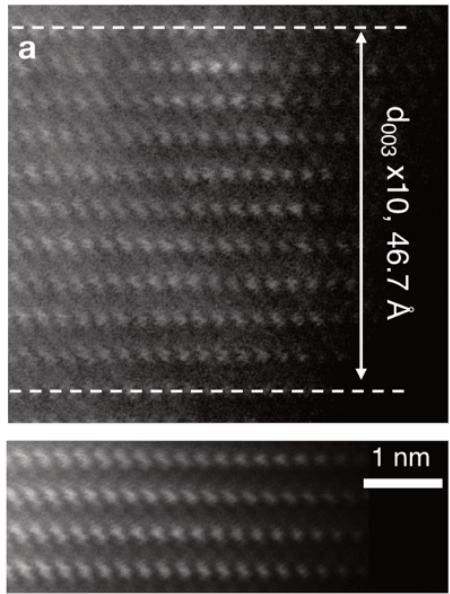

Aged, open channel

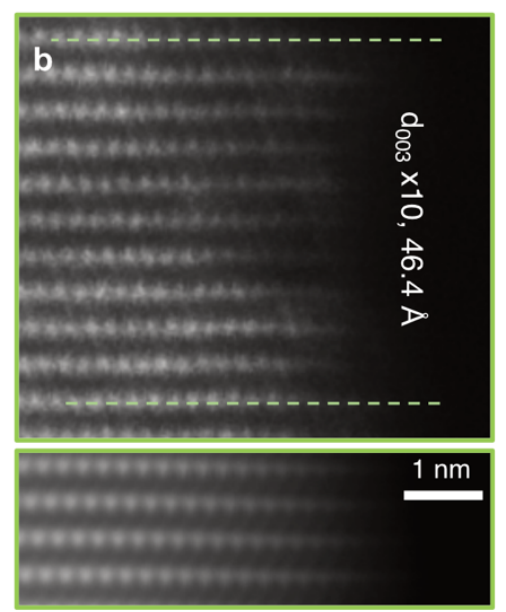

Aged, surface rock salt

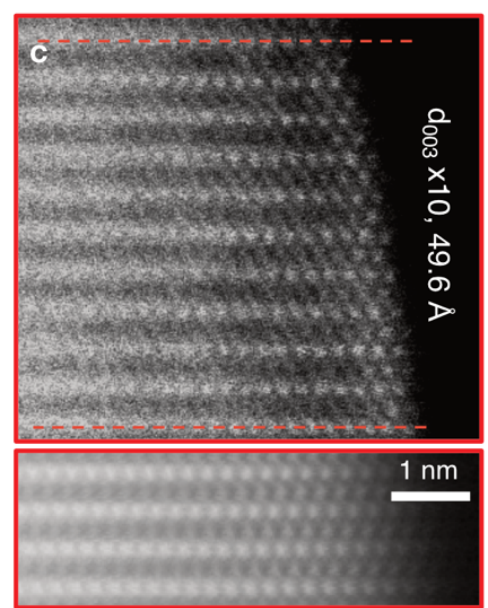

Figure 6. High- resolution STEM analysis of NMC811. DF images of pristine NMC811 (a), aged NMC showing an open lithium transfer channels (b) and with a rock salt surface reconstruction layer along the $<100>$ zone axis of the layered rhombohedral $R \overline{3} m$ structure (c). The images on the bottom raw show equivalent FFT-filtered to the top original images. The NMC sample for STEM imaging was cycled for 300 cycles, and then charged to a final SoC was at $4.3 \mathrm{~V}$ vs. Li.

Our model of the fatigue process is further supported by evidence from DF-STEM results (all along the $<100>$ zone axis of the layered rhombohedral $R \overline{3} m$ structure), which show a difference in the d spacing of the (003) plane of the layered structure in aged NMC particles at charged state (Figure 6). Considering that the difference in $\mathrm{d}$ spacing between the fatigued and active phases for a single layer is predicted to be small $(\sim 0.2 \AA$ based on the lattice parameters of the fatigued and active phases in the aged NMC after 300 cycles at $4.3 \mathrm{~V}$ shown in Figure 1 and Table S2). and below the instrumental resolution limit, we analysed the changes in spacings of a total of ten consecutive layers (more details in Supporting Information, Figure S16). As exemplified in Figure 6a, the width of this ten-layer slab for the pristine NMC811 prior to cycling was measured to be $46.7 \AA$, which is in line with the $c$ lattice parameter quantified from 
our XRD analysis of the pristine NMC811 (Table S1). Moreover, no obvious rock salt layer was found on the edge of the particle. A similar open channel structure was found on a particle from the aged sample after 300 cycles at a similar SoC (Figure 6b) and the spacing for ten layers of (003) planes was measured to be $46.4 \AA$ in this case. In contrast to these two scenarios, in which the particles show open channel structures at the surface, a discernibly larger spacing of $49.6 \AA$ was identified for the aged particle with a clear surface rock salt reconstruction on edge (Figure $6 \mathrm{c}$ ) and this leads to a net difference of $\sim 3 \AA$ between the aged NMC particles with and without surface rock salt layers.

The fact that the particle with a surface reconstruction layer has a larger $d$ spacing is indictive of a lower SoC for this particle. This is consistent with our model where particles with rock salt on the surface are proposed to be fatigued. We note that several possibilities, for instance, sample tilt, ${ }^{38}$ image drift, ${ }^{39}$ and thickness variation, could lead to errors in the determination of the lattice spacing from (S)TEM images. However, observation that the rock salt and layered phase have essentially identical d-spacings is independent of these errors. Furthermore, the quantitative difference we observed here that the particle with rock salt layers has a larger $\mathrm{d}$ spacing for the layered structure is robust. A detailed study with more rigorous statistical data and precise measurements is required to determine high-precision values for the layer spacings which is, however, beyond the scope of this paper.

This fatigue process is likely general in Ni-rich layered cathodes, particularly for those with the Ni content $\geq 80 \%$, as all these cathode materials exhibit both surface reconstruction as well as a large collapse in the unit cell at c direction at high SoCs. ${ }^{40}$ As a result, the lattice strain at the interface of the surface rock salt and layered structure in the bulk will build up at high SoCs. On electrochemical ageing, once the surface reconstruction layers become thick enough to pin the layered structure, the maximum accessible SoC becomes limited for that part of the cathode. Chemo-mechanical cracking caused by the large anisotropic lattice changes, likely to continue to occur in the more active (less fatigued) part of the cathode since it undergoes the largest changes in lattice parameters, will generate fresh surfaces where more rock salt phase can grow, leading to a further increase in the fraction of fatigued regions. In addition, although singlecrystal cathodes can efficiently mitigate the issue of inter-granular particle cracking, they are still susceptible to the fatigue degradation as a result of the surface reconstruction processes. The role of bulk dislocations and other faults that are often observed in these classes of materials on particle cracking and rock-salt formation is currently being explored. 


\section{Conclusions}

In conclusion, we show that Ni-rich NMC cathodes undergo a fatigue process, which results in a lowered accessible SoC at the end of charge, after repetitive cycling. The population of such fatigued cathode particles increases as ageing progresses, as quantified via the long-duration operando XRD experiments carried out in a novel laser-thinned coin cell design. Extensive analysis of the diffraction data reveals that the fatigued phases have the same and distinct structural characteristics. They can only be delithiated to a SoC of $\sim 75 \%$, which is associated with a lattice parameter $c / a$ ratio of $\sim 0.5111$. Neither a phase transformation of the bulk of the NMC cathode nor an increase in $\mathrm{Li} / \mathrm{Ni}$ antisite mixing was observed by diffraction; only small changes in the local structure and Li-ion mobility of the bulk were observed by ${ }^{7} \mathrm{Li}$ solid-state NMR spectroscopy. By examining single-crystal NMC materials, in which the fatigue degradation phenomenon was also observed, we show that the often-cited explanation in the literature, i.e. inter-granular cracking and kinetic limitations - which although they still occur in these materials, cannot be the primary origin of this structural fatigue.

Instead, for the first time, we propose that the primary cause of fatigue degradation in NMC is the high lattice mismatch at the interface of the layered structure in the bulk and the rock salt surface reconstruction layer when the cathode is at SoCs above the threshold of $\sim 75 \%$. For NMC811 at SoCs higher than $\sim 75 \%$, the unit cell contracts noticeably in both $c$ and $a / b$ directions, by $626 \%$ and $2 \%$, respectively, from $75 \%$ SoC to $90 \%$ SoC, resulting in significant deviations from the lattice constants of the rock salt. The NMC is expected to be electrochemically active between the fully lithiated state and $\sim 75 \% \mathrm{SoC}$ regardless of the presence of a rock salt surface layer as the lattice mismatch is relatively small within this SoC range. At SoCs higher than $\sim 75 \%$, the particles with a thick rock salt surface layer are prevented from further delithiation, consistent with both our operando long-duration X-ray diffraction and out ex-situ diffraction experiments.

We believe that this mechanism has been largely overlooked but is a critical part of the understanding of fast degradation of the Ni-rich layered cathodes. With the same upper cut-off voltage, higher Ni-content NMCs show more significant lattice parameter change, particularly the collapse of the $c$ parameter, as compared to the lower Ni-content analogous, making the Ni-rich layer cathodes more susceptible to the fatigue degradation mechanism. Our findings highlight the urgent need for developing effective countermeasures, for instance, uniform protective coatings and functional electrolyte additives, to improve the performance of Ni-rich cathode materials. 


\section{Acknowledgement:}

The authors are grateful to K. Kleiner, K. J. Griffith and W. Wei for their support with the longduration X-ray diffraction experiments, and J. Morzy and J. Lu for assisting in the TEM experiments. C.X. is grateful to H. Liu for discussions on the X-ray diffraction data analysis. The authors are grateful for funding support from the ISCF Faraday Challenge Fast Start project on "Degradation of Battery Materials" made available through grant EP/S003053/1. C.X. acknowledges support from a Science and Technology Facilities Council (STFC) Experimental Design Award from the STFC Batteries Network (ST/R006873/1). 


\section{References}

1. Tarascon, J. M. \& Armand, M. Issues and challenges facing rechargeable lithium batteries. Nature 414, 359-367 (2001).

2. Blomgren, G. E. The Development and Future of Lithium Ion Batteries. J. Electrochem. Soc. 164, A5019-A5025 (2016).

3. Kim, J. et al. Prospect and Reality of Ni-Rich Cathode for Commercialization. $A d v$. Energy Mater. 8, 1702028 (2018).

4. Myung, S.-T. et al. Nickel-Rich Layered Cathode Materials for Automotive Lithium-Ion Batteries: Achievements and Perspectives. ACS Energy Letters 2, 196-223 (2017).

5. Li, W., Erickson, E. M. \& Manthiram, A. High-nickel layered oxide cathodes for lithiumbased automotive batteries. Nat. Energy 5, 26-34 (2020).

6. Noh, H.-J., Youn, S., Yoon, C. S. \& Sun, Y.-K. Comparison of the structural and electrochemical properties of layered $\mathrm{Li}\left[\mathrm{Ni}_{\mathrm{x}} \mathrm{Co}_{\mathrm{y}} \mathrm{Mn}_{\mathrm{z}}\right] \mathrm{O}_{2}(\mathrm{x}=1 / 3,0.5,0.6,0.7,0.8$ and 0.85) cathode material for lithium-ion batteries. J. Power Sources 233, 121-130 (2013).

7. Jung, R., Metzger, M., Maglia, F., Stinner, C. \& Gasteiger, H. A. Oxygen Release and Its Effect on the Cycling Stability of $\mathrm{LiNi}_{\mathrm{x}} \mathrm{Mn}_{\mathrm{y}} \mathrm{Co}_{\mathrm{z}} \mathrm{O}_{2}(\mathrm{NMC})$ Cathode Materials for Li-Ion Batteries. J. Electrochem. Soc. 164, A1361-A1377 (2017).

8. Ryu, H.-H., Park, K.-J., Yoon, C. S. \& Sun, Y.-K. Capacity Fading of Ni-Rich $\mathrm{Li}\left[\mathrm{Ni}_{\mathrm{x}} \mathrm{Co}_{\mathrm{y}} \mathrm{Mn}_{1-\mathrm{x}-\mathrm{y}}\right] \mathrm{O}_{2}(0.6 \leq \mathrm{x} \leq 0.95)$ Cathodes for High-Energy-Density Lithium-Ion Batteries: Bulk or Surface Degradation. Chem. Mater. 30, 1155-1163 (2018).

9. Friedrich, F. et al. Editors' Choice-Capacity Fading Mechanisms of NCM-811 Cathodes in Lithium-Ion Batteries Studied by X-ray Diffraction and Other Diagnostics. J. Electrochem. Soc. 166, A3760-A3774 (2019).

10. de Biasi, L. et al. Chemical, Structural, and Electronic Aspects of Formation and Degradation Behavior on Different Length Scales of Ni-Rich NCM and Li-Rich HENCM Cathode Materials in Li-Ion Batteries. Adv. Mater. 0, 1900985 (2019).

11. Kondrakov, A. O. et al. Anisotropic Lattice Strain and Mechanical Degradation of Highand Low-Nickel NCM Cathode Materials for Li-Ion Batteries. J. Phys. Chem. C 121, 3286-3294 (2017).

12. Märker, K., Reeves, P. J., Xu, C., Griffith, K. J. \& Grey, C. P. Evolution of Structure and Lithium Dynamics in $\mathrm{LiNi}_{0.8} \mathrm{Mn}_{0.1} \mathrm{Co}_{0.1} \mathrm{O}_{2}$ (NMC811) Cathodes during Electrochemical Cycling. Chem. Mater. 31, 2545-2554 (2019).

13. Lin, F. et al. Profiling the nanoscale gradient in stoichiometric layered cathode particles for lithium-ion batteries. Energy Environ. Sci. 7, 3077 (2014).

14. Lin, F. et al. Surface reconstruction and chemical evolution of stoichiometric layered cathode materials for lithium-ion batteries. Nat Commun 5, 3529 (2014).

15. Xiao, P., Shi, T., Huang, W. \& Ceder, G. Understanding Surface Densified Phases in NiRich Layered Compounds. ACS Energy Letters 4, 811-818 (2019).

16. Tian, C., Lin, F. \& Doeff, M. M. Electrochemical Characteristics of Layered Transition Metal Oxide Cathode Materials for Lithium Ion Batteries: Surface, Bulk Behavior, and Thermal Properties. Acc. Chem. Res. 51, 89-96 (2018). 
17. Radin, M. D. et al. Narrowing the Gap between Theoretical and Practical Capacities in Li-Ion Layered Oxide Cathode Materials. Adv. Energy Mater. 7, 1602888 (2017).

18. $\mathrm{Yu}, \mathrm{Y}$. et al. Coupled $\mathrm{LiPF}_{6}$ Decomposition and Carbonate Dehydrogenation Enhanced by Highly Covalent Metal Oxides in High-Energy Li-Ion Batteries. J. Phys. Chem. C 122, 27368-27382 (2018).

19. Zhang, Y. et al. Revealing electrolyte oxidation via carbonate dehydrogenation on Nibased oxides in Li-ion batteries by in situ Fourier transform infrared spectroscopy. Energy Environ. Sci. 13, 183-199 (2020).

20. Delmas, C., Fouassier, C. \& Hagenmuller, P. Structural classification and properties of the layered oxides. Physica $B+C$ 99, 81-85 (1980).

21. de Biasi, L. et al. Phase Transformation Behavior and Stability of $\mathrm{LiNiO}_{2}$ Cathode Material for Li-Ion Batteries Obtained from In Situ Gas Analysis and Operando X-Ray Diffraction. ChemSusChem 12, 2240-2250 (2019).

22. LI, W., REIMERS, J. \& DAHN, J. In situ X-ray diffraction and electrochemical studies of $\mathrm{Li}_{1-\mathrm{x}} \mathrm{NiO}_{2}$. Solid State Ionics 67, 123-130 (1993).

23. Li, H., Zhang, N., Li, J. \& Dahn, J. R. Updating the Structure and Electrochemistry of $\mathrm{Li}_{\mathrm{x}} \mathrm{NiO}_{2}$ for $0 \leq \mathrm{x} \leq 1$. J. Electrochem. Soc. 165, A2985-A2993 (2018).

24. Li, W. et al. Mn versus Al in Layered Oxide Cathodes in Lithium-Ion Batteries: A Comprehensive Evaluation on Long-Term Cyclability. Adv. Energy Mater. 8, 1703154 (2018).

25. Kleiner, K. et al. Fatigue of $\mathrm{LiNi}_{0.8} \mathrm{Co}_{0.15} \mathrm{Al}_{0.05} \mathrm{O}_{2}$ in commercial Li ion batteries. J. Power Sources 273, 70-82 (2015).

26. Liu, H. et al. Intergranular Cracking as a Major Cause of Long-Term Capacity Fading of Layered Cathodes. Nano Lett 17, 3452-3457 (2017).

27. Long, B. R. et al. Enabling High-Energy, High-Voltage Lithium-Ion Cells: Standardization of Coin-Cell Assembly, Electrochemical Testing, and Evaluation of Full Cells. J. Electrochem. Soc. 163, A2999-A3009 (2016).

28. Murray, C. A. et al. New synchrotron powder diffraction facility for long-duration experiments. J. Appl. Crystallogr. 50, 172-183 (2017).

29. Bielecki, A. \& Burum, D. P. Temperature Dependence of 207 Pb MAS Spectra of Solid Lead Nitrate. An Accurate, Sensitive Thermometer for Variable-Temperature MAS. Journal of Magnetic Resonance, Series A 116, 215-220 (1995).

30. Schweidler, S. et al. Investigation into Mechanical Degradation and Fatigue of High-Ni NCM Cathode Material: A Long-Term Cycling Study of Full Cells. ACS Applied Energy Materials 2, 7375-7384 (2019).

31. Li, J., Downie, L. E., Ma, L., Qiu, W. \& Dahn, J. R. Study of the Failure Mechanisms of $\mathrm{LiNi}_{0.8} \mathrm{Mn}_{0.1} \mathrm{Co}_{0.1} \mathrm{O}_{2}$ Cathode Material for Lithium Ion Batteries. J. Electrochem. Soc. 162, A1401-A1408 (2015).

32. Gilbert, J. A. et al. Cycling Behavior of NCM523/Graphite Lithium-Ion Cells in the 34.4 V Range: Diagnostic Studies of Full Cells and Harvested Electrodes. J. Electrochem. Soc. 164, A6054-A6065 (2016). 
33. Makimura, Y. et al. Factors affecting cycling life of $\mathrm{LiNi}_{0.8} \mathrm{Co}_{0.15} \mathrm{Al}_{0.05} \mathrm{O}_{2}$ for lithium-ion batteries. J. Mater. Chem. A 4, 8350-8358 (2016).

34. Grey, C. P. \& Dupré, N. NMR Studies of Cathode Materials for Lithium-Ion Rechargeable Batteries. Chem. Rev. 104, 4493-4512 (2004).

35. Pell, A. J., Pintacuda, G. \& Grey, C. P. Paramagnetic NMR in solution and the solid state. Prog. Nucl. Magn. Reson. Spectrosc. 111, 1-271 (2019).

36. Liu, $H$. et al. Identifying the chemical and structural irreversibility in $\mathrm{LiNi}_{0.8} \mathrm{Co}_{0.15} \mathrm{Al}_{0.05} \mathrm{O}_{2}$ - a model compound for classical layered intercalation. Journal of Materials Chemistry A 6, 4189-4198 (2018).

37. Hwang, S. et al. Investigation of Changes in the Surface Structure of $\mathrm{Li}_{\mathrm{x}} \mathrm{Ni}_{0.8} \mathrm{Co}_{0.15} \mathrm{Al}_{0.05} \mathrm{O}_{2}$ Cathode Materials Induced by the Initial Charge. Chem. Mater. 26, 1084-1092 (2014).

38. Zhou, D. et al. Sample tilt effects on atom column position determination in ABF-STEM imaging. Ultramicroscopy 160, 110-117 (2016).

39. Ophus, C., Ciston, J. \& Nelson, C. T. Correcting nonlinear drift distortion of scanning probe and scanning transmission electron microscopies from image pairs with orthogonal scan directions. Ultramicroscopy 162, 1-9 (2016).

40. Goonetilleke, D. et al. Structural Evolution and High-Voltage Structural Stability of $\mathrm{Li}\left(\mathrm{Ni}_{\mathrm{x}} \mathrm{Mn}_{\mathrm{y}} \mathrm{Co}_{\mathrm{z}}\right) \mathrm{O}_{2}$ Electrodes. Chem. Mater. 31, 376-386 (2019). 


\section{Supplementary information}

\section{Bulk fatigue induced by surface reconstruction in layered Ni-rich oxide cathodes for Li-ion batteries}

Chao Xu, ${ }^{1,2}$ Katharina Märker, ${ }^{1,2}$ Juhan Lee, ${ }^{2,3}$ Amoghavarsha Mahadevegowda, ${ }^{2,4}$ Philip J. Reeves, ${ }^{1,2}$ Sarah Day, ${ }^{5}$ Matthias F. Groh, ${ }^{1}$ Steffen Emge, ${ }^{1}$ Caterina Ducati, ${ }^{2,4}$ B. Layla Mehdi, ${ }^{2,3}$ Chiu Tang, ${ }^{5}$ Clare P. Grey ${ }^{*}, 1,2$

${ }^{1}$ Department of Chemistry, University of Cambridge, Lensfield Road, Cambridge CB2 1EW, United Kingdom

2 The Faraday Institution, Quad One, Harwell Science and Innovation Campus, Didcot OX11 ORA, United Kingdom

${ }^{3}$ Department of Mechanical, Materials and Aerospace Engineering, University of Liverpool, Liverpool, L69 3GH, United Kingdom

${ }^{4}$ Department of Materials Science and Metallurgy, University of Cambridge, 27 Charles Babbage Road, Cambridge CB3 OFS, United Kingdom

${ }^{5}$ Diamond Light Source, Harwell Science and Innovation Campus, Didcot, UK 


\section{Table of Contents}

Table S1: Rietveld refinement results of the pristine polycrystalline NMC811 ............................................

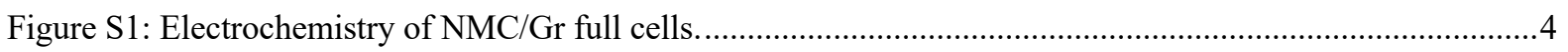

Figure S2: Ex-situ Laboratory PXRD pattern of aged NMC at a discharged state .........................................5

Discussion: choice of structural model for Rietveld refinement ................................................................

Table S2: Rietveld refinement results of the electrochemically aged polycrystalline NMC811 at charged state...7

Figure S3: Peak assignment of PXRD patterns of LDE coin cell..............................................................

Figure S4: Evolution of the lattice parameters of the NMC cathode during the charging process of cycle 348,

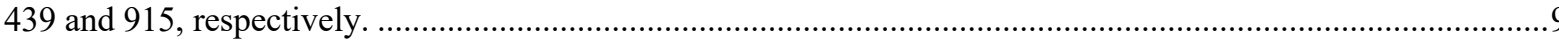

Figure S5: Evolution of the (113) NMC $_{\mathrm{N}}$ reflection during long-term electrochemical ageing............................. 10

Figure S6: Diffraction results of the NMC cathode after 1200 cycles at $4.3 \mathrm{~V}$ vs. Li.....................................11

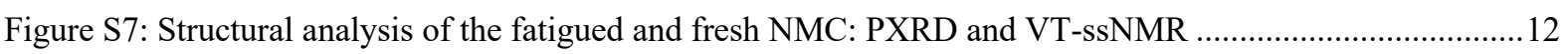

Figure S8: Experimental and calculated 7Li ssNMR spectra at variable temperatures...................................13

Figure S9: Calculated Li hopping rates and fraction of the high mobility component from VT NMR results.....15

Figure S10: ${ }^{7} \mathrm{Li}$ NMR spectra recorded as rotor synchronised Hahn-echoes with an increasing number of rotor

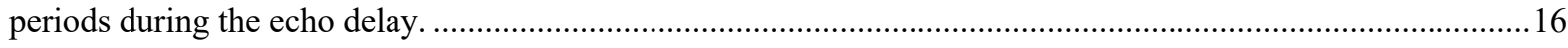

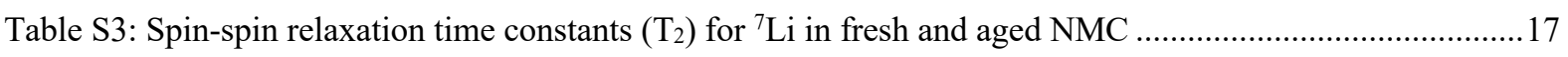

Figure S11: ${ }^{7} \mathrm{Li}$ ssNMR spectra of an aged NMC cathodes after 1200 cycles at $4.3 \mathrm{~V}$ vs. Li. .........................18

Figure S12. (S)TEM images of the surface of an aged NMC811 particle...................................................20

Figure S13: High resolution TEM images of the formation of surface reconstruction layer ............................21

Figure S14: Single-crystal NMC: diffraction pattern and electrochemical performance................................22

Table S4: Rietveld refinement result of the pristine single-crystal NMC . ...................................................23

Figure S15: X-ray diffraction pattern of aged single-crystal NMC at a charged state...................................24

Figure S16. Analysis of the d003 spacing for the pristine and aged NMC811 particles..................................25

References .26 
Table S1: Rietveld refinement results of the pristine polycrystalline NMC811.

Table S1. Summary of refinement results against PXRD of the pristine NMC811

\begin{tabular}{llllll}
\hline Site & Atom & $\mathrm{x}$ & $\mathrm{y}$ & $\mathrm{z}$ & Occupancy \\
\hline $3 \mathrm{a}$ & $\mathrm{Ni}$ & 0 & 0 & 0 & $0.789(1)$ \\
& $\mathrm{Mn}$ & 0 & 0 & 0 & 0.1 \\
& $\mathrm{Co}$ & 0 & 0 & 0 & 0.1 \\
& $\mathrm{Li}$ & 0 & 0 & 0 & $0.011(1)$ \\
$3 \mathrm{~b}$ & $\mathrm{Li}$ & 0 & 0 & $1 / 2$ & $0.989(1)$ \\
& $\mathrm{Ni}$ & 0 & 0 & $1 / 2$ & $0.011(1)$ \\
$6 \mathrm{c}$ & $\mathrm{O}$ & 0 & 0 & $0.2584(2)$ & 1 \\
\hline
\end{tabular}

Space group: $R \overline{3} m$

Fitting quality: $R_{\mathrm{wp}}=5.501, \mathrm{R}_{\exp }=3.125$, goodness of fit $=1.760$

Lattice parameters: $a=b=2.87209(5) \AA, c=14.2008(3) \AA$ 
Figure S1: Electrochemistry of NMC/Gr full cells.
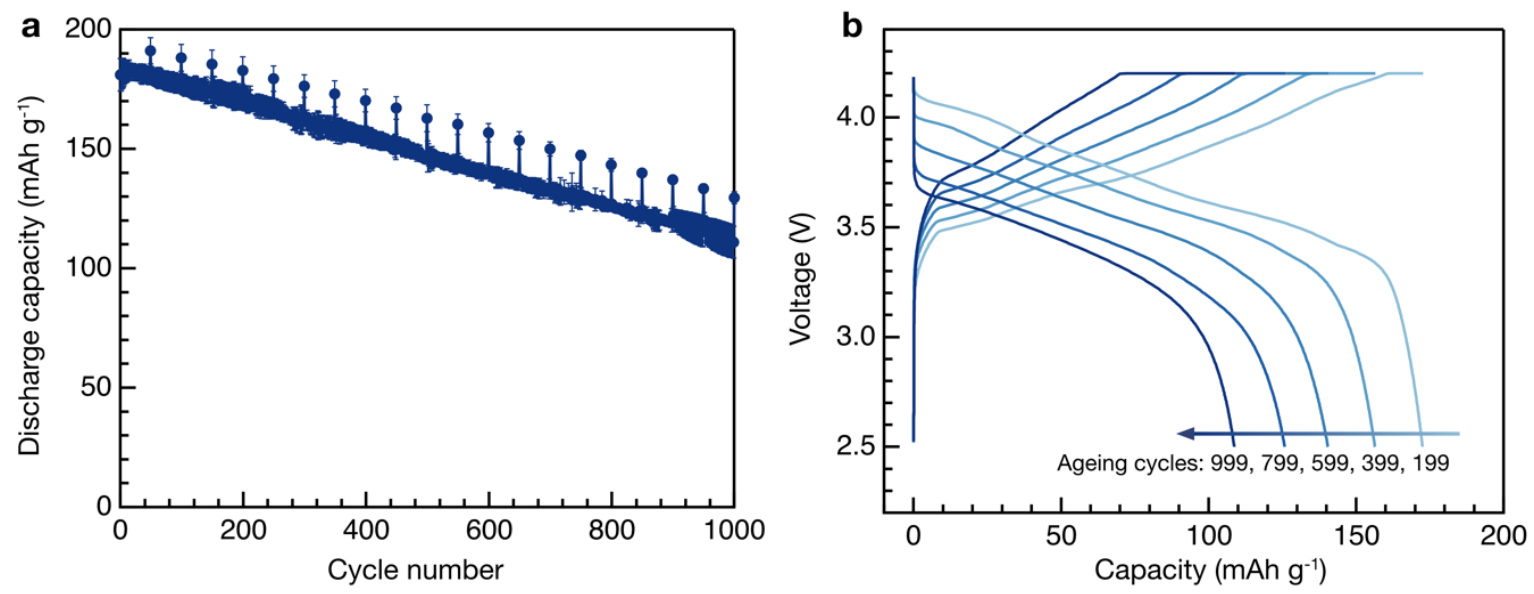

Figure S1. Long-term cyclability of NMC811/graphite full-cells. (a) Capacity retention of an average of two cells. The spikes in discharge capacity is attributed to the slow diagnostic cycles (C/20 rate) performed once every 50 ageing cycles. (b) Voltage profiles of a NMC811/graphite full-cell at ageing cycles of 199, 399, 599, 799 and 999. These cycles are all performed at the same rate of $\mathrm{C} / 2$. 
Figure S2: Ex-situ Laboratory PXRD pattern of aged NMC at a discharged state

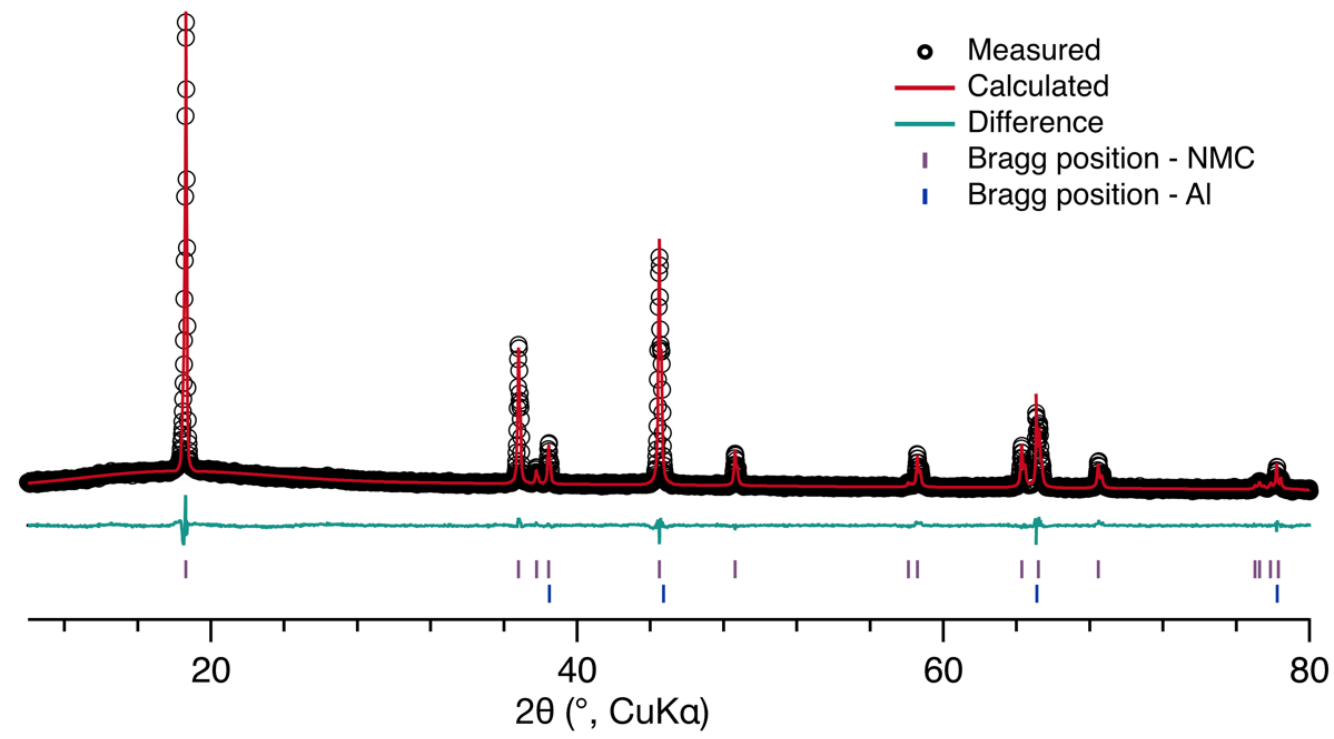

Figure S2. XRD pattern of NMC after 300 cycles at a discharged state. Lattice parameters from refinement: $a=b=2.86003(3) \AA, c=14.2797(3) \AA$. The NMC electrode was aged in an $\mathrm{NMC/graphite} \mathrm{full-cell} \mathrm{for} 300$ cycles, and reassembled into a $\mathrm{NMC/Li}$ graphite cell and discharged to $3.0 \mathrm{~V}$ to reach the fully lithiated state. 


\section{Discussion: choice of structural model for Rietveld refinement}

Potential origins of the observed peak splitting:

1. Symmetry reduction due to structural distortion. Collective Jahn-Teller distortion and/or lithium vacancy ordering in layered lithium transition metal (TM) oxides can distort the rhombohedral $R \overline{3} m$ structure to monoclinic $\mathrm{C} 2 / \mathrm{m}$. For instance, $\mathrm{LiNiO}_{2}$ undergoes a $\mathrm{O} 3$ to monoclinic structural transformation at $\sim 3.65-3.8 \mathrm{~V}\left(\mathrm{vs} . \mathrm{Li} / \mathrm{Li}^{+}\right)$due to the Jahn-Teller activity of the $\mathrm{Ni}^{3+}$ ions and the ordering of $\mathrm{Li}^{+}$ions. ${ }^{1,2}$ However, this is unlikely to be reason for the phase segregation we observed in this work since the transformation from rhombohedral to monoclinic should not split the $(00 l)$ reflection (i.e. $(h k l)$ reflections where $h=k=0){ }^{2}$ Furthermore, this structural distortion is observed at low states of charge.

2. Structural transformation from layered hexagonal to spinel and/or distorted rock salt. It is well established that delithiated layered oxides are thermodynamically less stable compared to spinel and rock salt phases. ${ }^{1,3}$ The reason why many oxide materials still can maintain the layered structure upon lithium extraction and reinsertion is that the migration of transition metal ions from the TM layer to the octahedral sites in the lithium layer, which is required to transform from layered to spinel and/or rock salt, is kinetically limited. Moreover, the ageing conditions used in the present study are considered to be rather mild: reasonable rate, low upper cutoff voltage and room temperature, so we believe that spinel and/or rock salt phases are unlikely to form in the bulk of the NMC material. This hypothesis is further supported by the fact that no obvious increase in $\mathrm{Li} / \mathrm{Ni}$ antisite mixing observed in electrochemically aged cathodes at discharged state (more details are given in the section of Long-range structure and Li local environments) Even such phase transformation did occur, considering the fact that no spinel nor rock salt phases are observed at discharged state (Figure S2), this will require a reverse transformation from spinel and/or rock salt back to layered, which is both thermodynamically unfavored as well as kinetically limited. Therefore, we believe that the phase segregation is not due to bulk structure phase transformation to spinel/rock salt.

Therefore, we analysed the diffraction result using multiple rhombohedral R-3m phases, which represents NMC at different SOCs. This is also supported by the other works which investigated similar reaction heterogeneity in NCA and NMC at high SOCs after long-term cycling. ${ }^{4-7}$ 
Table S2: Rietveld refinement results of the electrochemically aged polycrystalline NMC811 at charged state.

Table S2. Summary of refinement results of the electrochemically aged polycrystalline NMC811 at charged state $\left(4.3 \mathrm{~V}\right.$ vs. $\left.\mathrm{Li}^{-} \mathrm{Li}^{+}\right)$. The corresponding diffraction pattern is shown in Figure 1c.

\begin{tabular}{llll}
\hline & Fatigued & Intermediate & Active \\
\hline Phase fraction $(\mathrm{wt} \%)$ & $21(2)$ & $25(2)$ & $54(2)$ \\
$a(\AA)$ & $2.8203(3)$ & $2.8174(2)$ & $2.8169(9)$ \\
$c(\AA)$ & $14.416(1)$ & $14.265(6)$ & $14.042(2)$ \\
Volume $\left(\AA^{3}\right)$ & $99.30(2)$ & $98.07(4)$ & $96.50(2)$ \\
Li occupancy & $0.255(1)$ & $0.201(1)$ & $0.1502(4)$ \\
\hline
\end{tabular}

Fitting quality: $R_{w p}=2.981, R_{\exp }=3.079$, goodness of fit $=0.968$ 
Figure S3: Peak assignment of PXRD patterns of LDE coin cell.
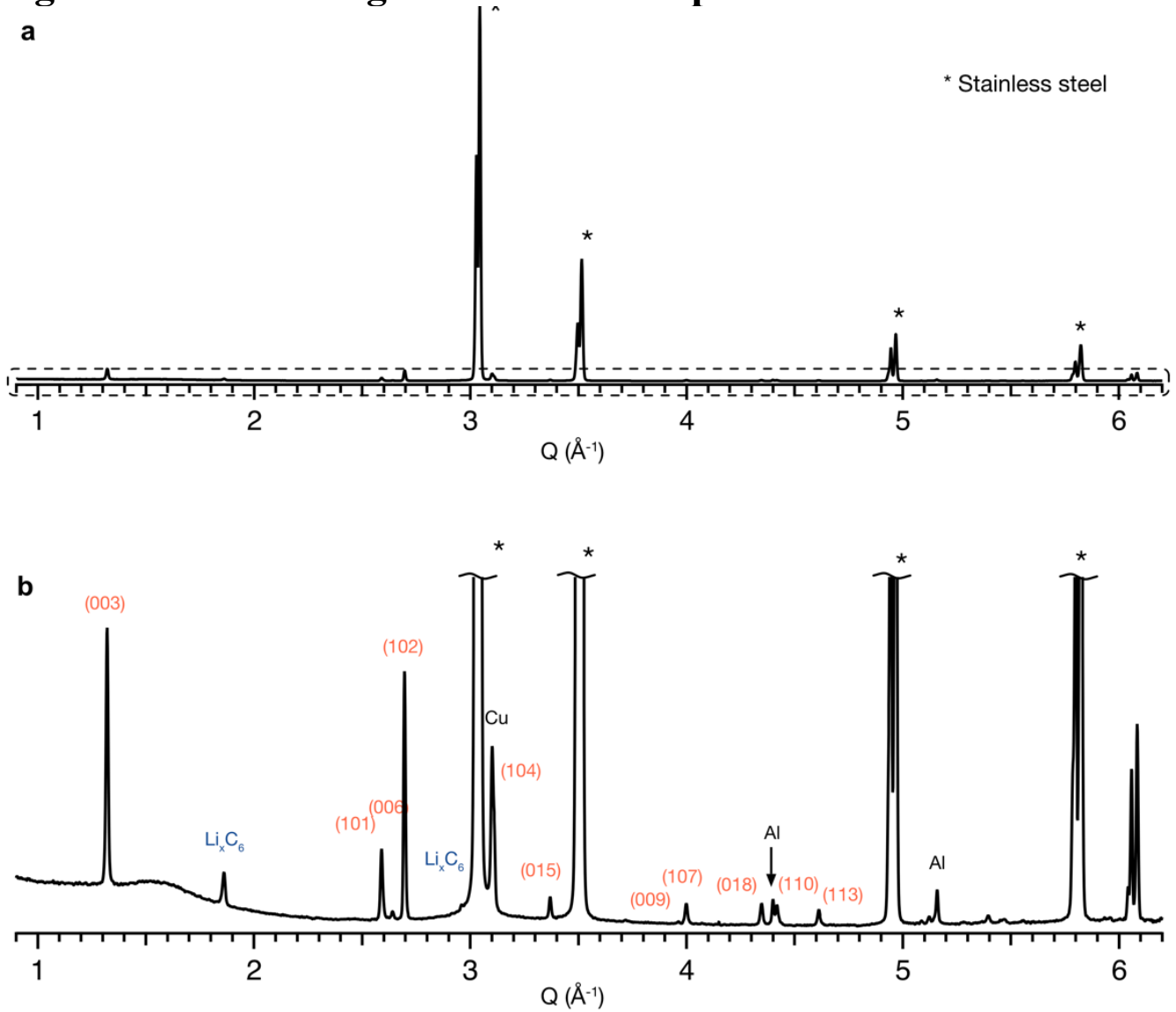

Figure S3. (a) Diffraction pattern of the LDE coin cell at full intensity scale. (b) Zoomed so as to index the diffraction peaks of the active materials. NMC reflection are marked in red and graphite/lithiated graphite are marked in blue 
Figure S4: Evolution of the lattice parameters of the NMC cathode during the charging process of cycle 348,439 and 915 , respectively.
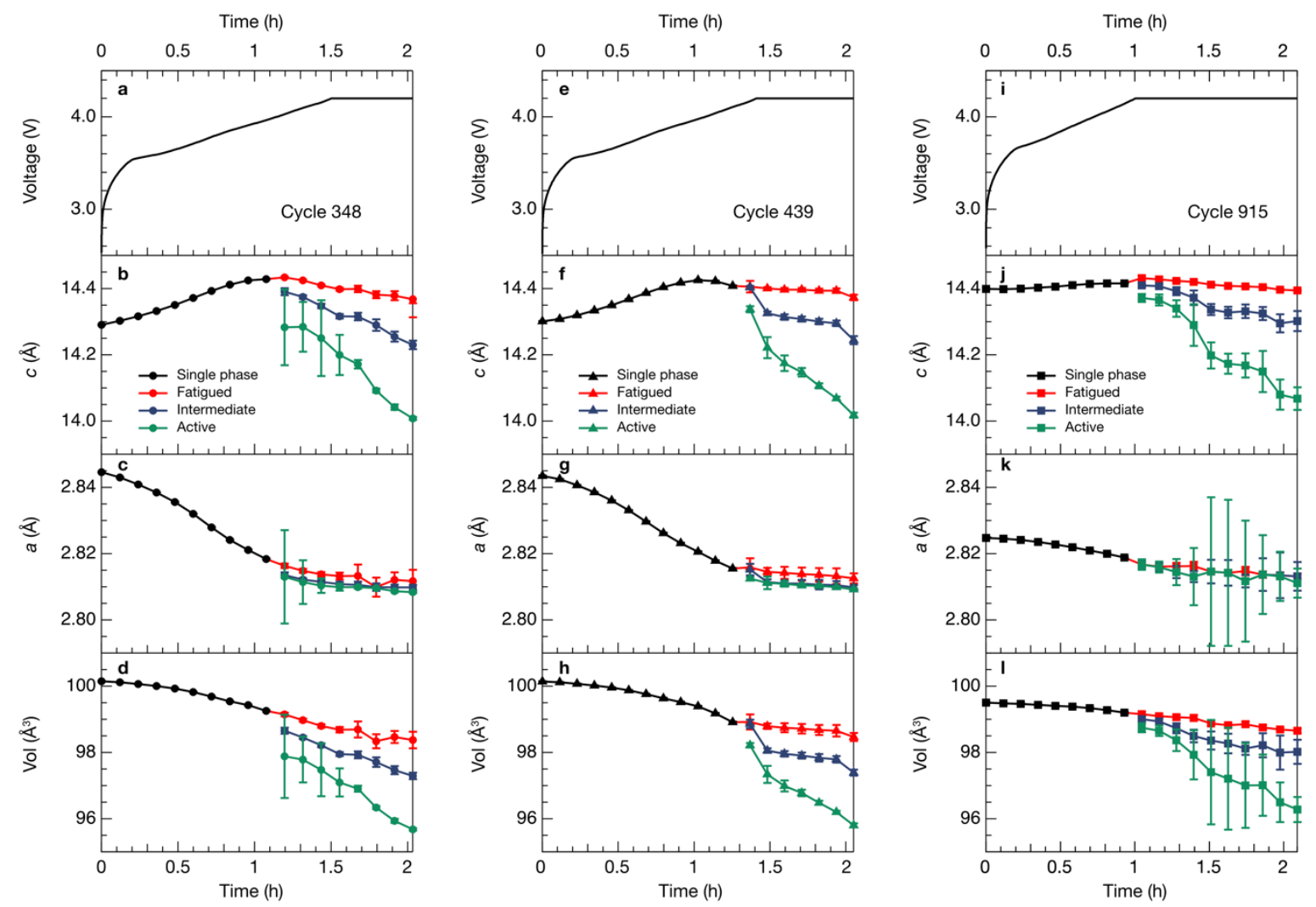

Figure S4. Evolution of the lattice parameters obtained from refinement against the LDE diffraction pattern. (a-d) voltage profile, lattice parameters $c, a$ and unit cell volume of the NMC cathode in cycle 348; (e-h) voltage profile, lattice parameters $c, a$ and unit cell volume of the NMC cathode in cycle 439; (i-1) voltage profile, lattice parameters $c, a$ and unit cell volume of the NMC cathode in cycle 91 


\section{Figure S5: Evolution of the (113) $)_{\mathrm{NMC}}$ reflection during long-term electrochemical ageing.}

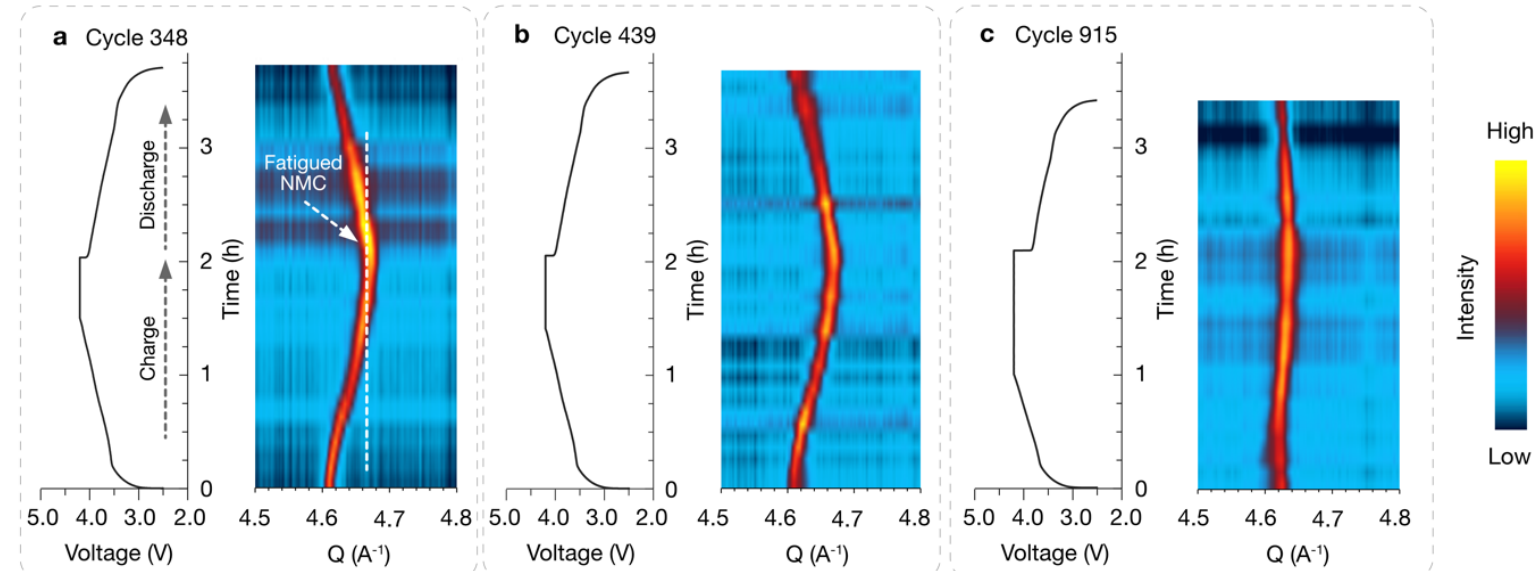

Figure S5. Evolution of the (113) NMC $_{\mathrm{N}}$ reflections during cycle (a) 348, (b) 439 and (c) 915.

Discussion: the (113) reflection changes monotonically as a function of $\mathrm{SoC}$ and therefore is an excellent indication of the state of charge for a full charge and discharge. If the fatigued phase were completely inactive on charge and discharge, a distinct (113) signal with fixed peak position and intensity should be observed. However, in Figure S6(a) for instance, the (113) signal during cycle 348 gradually moved to higher Q on charge and back to lower on discharge reversibly. Therefore, the fatigued phase is still electrochemically active during the lower SoC regime but the maximum accessible $\mathrm{SoC}$ is limited and lower compared to the rest of the active material. 
Figure S6: Diffraction results of the NMC cathode after 1200 cycles at 4.3 V vs. Li.

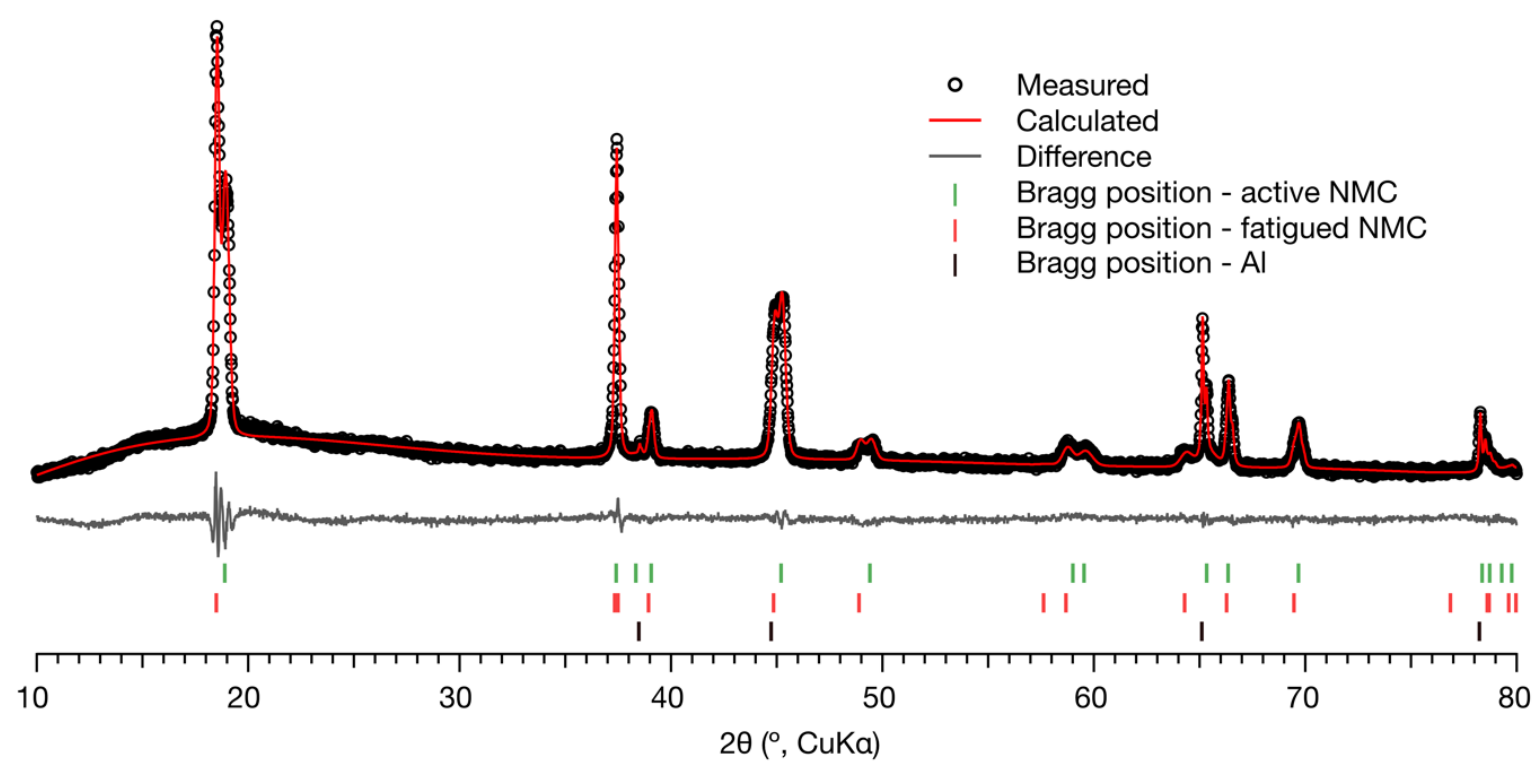

Figure S6. XRD pattern of the NMC cathode at $4.3 \mathrm{~V}$ vs. Li after ageing 1200 cycles in LDE coin cell. The electrode was extracted from the LDE cell after ageing and re-assembled into a half cell with Li metal. The half-cell was then charged at a rate of $\mathrm{C} / 20$ with a voltage hold step at $4.3 \mathrm{~V}$ until current drops below $\mathrm{C} / 1000$.

Discussion: due to the poor quality of the pattern which was obtained from a laboratory diffractometer as compared to that from synchrotron, we were unable to resolve the intermediate phase and the Rietveld refinement was carried out using two rhombohedral phases. The obtained refinement results are: (1) active phase: phase fraction $=53(1) \%, a=b$ $=2.8158(2) \AA, c=14.080(2) \AA, \mathrm{SoC} \approx 84 \%$; (2) fatigued phase: phase fraction $=47(1) \%, a$ $=b=2.8186(2) \AA, c=14.386(2) \AA, \mathrm{SoC} \approx 76 \%$. 
Figure S7: Structural analysis of the fatigued and fresh NMC: PXRD and VT-sSNMR
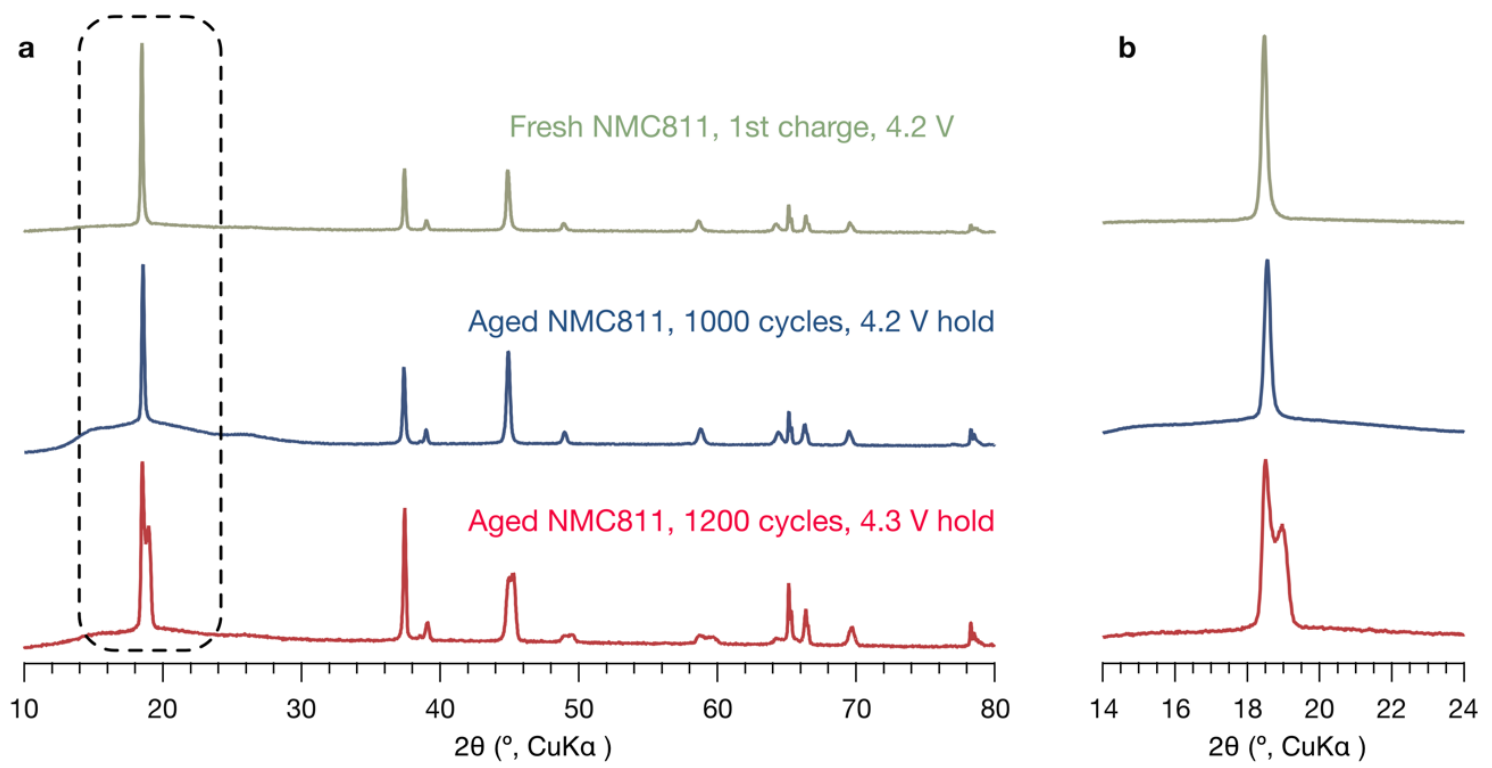

Figure S7. Structural analysis of the fatigued and fresh NMC. (a) X-ray diffraction pattern of the fresh NMC811 at 4.2 V, and aged NMC811 at $4.2 \mathrm{~V}$ and $4.3 \mathrm{~V}$. (b) Expanded (003) region. 


\section{Figure S8: Experimental and calculated 7Li ssNMR spectra at variable temperatures}

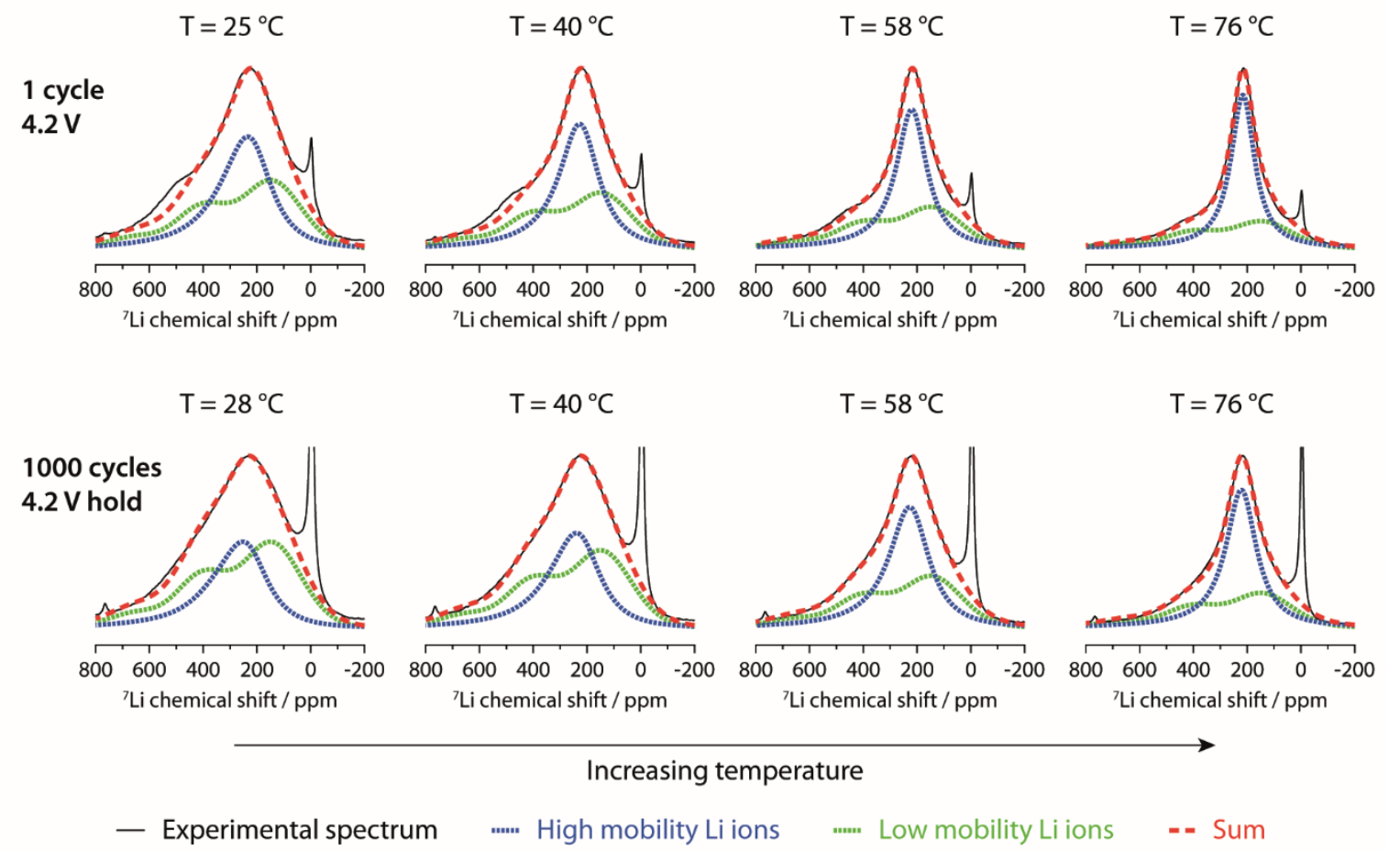

Figure S8. Comparisons of modelled (red dashed line) and experimental (black solid line) ${ }^{7} \mathrm{Li}$ variable temperature (VT) NMR spectra for "fresh" NMC811 at $4.2 \mathrm{~V}$ (top) and aged NMC811 held at $4.2 \mathrm{~V}$ (bottom). In all cases a motionally averaged sharp component corresponding to Li ions with high mobility (blue dotted lines) and a non-averaged component corresponding to $\mathrm{Li}$ ions with lower mobility (green dotted lines) were required to produce good agreement between experiment and calculation. The Li hopping rates of the high mobility component as well as its fraction of the total signal integral are plotted in Figure S8.

\section{Discussion:}

The VT NMR spectra were modelled following the same approach we described earlier for lower SoC samples of NMC811.8 They are approximated as the sum (red) of a spectrum due to high mobility Li ions (blue) and a spectrum due to lower mobility Li ions (green). As described in the paper, we define high mobility ions as having a hopping rate fast enough to induce narrowing of the NMR signal $(>20 \mathrm{kHz})$. The calculation of these two spectra is outlined below.

The spectrum for low mobility Li ions was calculated using a random solution model which assumes that both $\mathrm{TM}$ and $\mathrm{Li}$ ions are distributed randomly within their respective layers. We assumed $75 \%$ delithiation of NMC811 for both samples and the following oxidation states of the TM ions: $\mathrm{Ni}^{3.65+}, \mathrm{Mn}^{4+}, \mathrm{Co}^{3+}$. The spectrum is then calculated by summing the spectra for all possible Li environments, with each single spectrum being computed based on the probability and the chemical shift of the respective environment. The low mobility spectrum was shifted by $80 \mathrm{ppm}$ to higher ppm values in all spectra in Figure S7 to improve the agreement between experimental and calculated spectra. 
The spectrum for high mobility Li ions was calculated using Norris' formula to account for motional averaging. 'It allows fast calculation of spectral lineshapes for hopping between multiple sites and is based on the assumption that hopping occurs between all sites with one uniform hopping rate. For these calculations, the probabilities and chemical shifts of the different Li environments were used as determined before by the random solution model. Norris' formula also requires the linewidth of a single Li site without exchange $\left(v_{1 / 2, \text { no-exch }}\right)$. As this value is hard to determine experimentally, we used $100 \mathrm{~Hz}, 1000 \mathrm{~Hz}$, and $2000 \mathrm{~Hz}$, analogous to our previous work. .

Both spectra were added for modeling the experimental spectra. The following parameters were varied to achieve a satisfactory agreement between the model and experiment: The hopping rate, the ratio of the low and high mobility spectra, and the overall shift of the high mobility spectrum. The resulting plot of experimental and calculated spectra is shown in Figure S7. Figure S8 presents some parameters used for the calculations: The Li hopping rates for

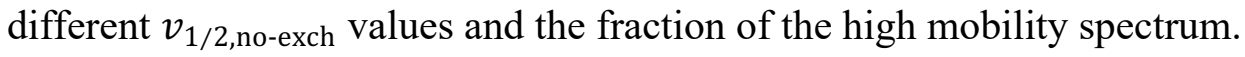


Figure S9: Calculated Li hopping rates and fraction of the high mobility component from VT NMR results.
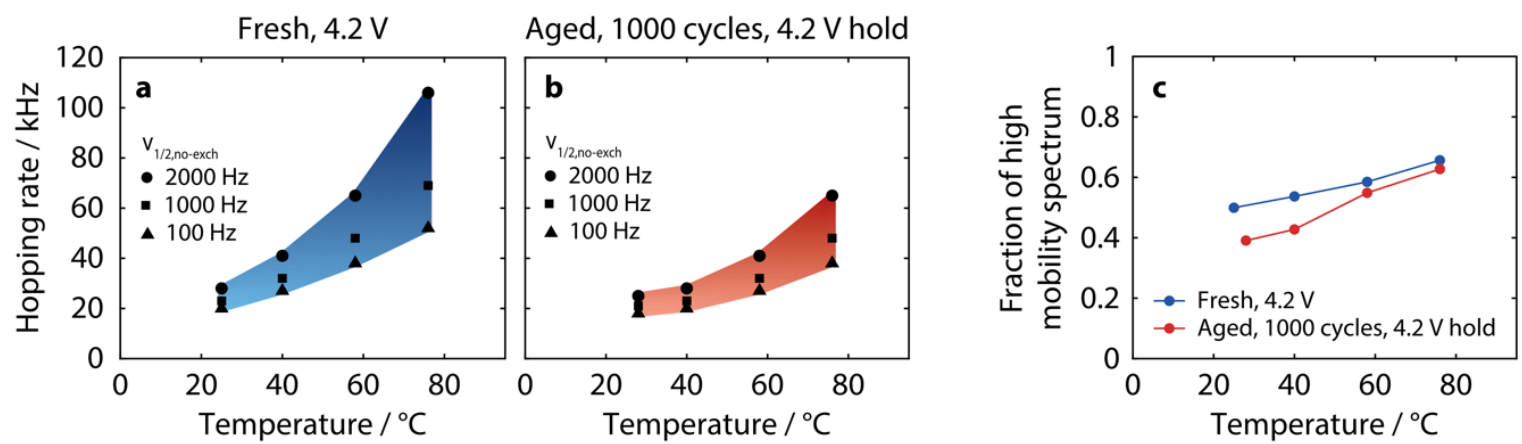

Figure S9. Parameters used for modelling the spectra shown in Figure S7. (a,b) Calculated hopping rates for the high mobility signal component for fresh (a) and aged (b) NMC811

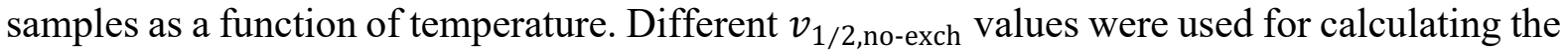
hopping rates (see legend in plot). Blue and red shading have been added to the plot for illustrational purposes. (c) Proportion of the high mobility component for fresh (blue) and aged (red) NMC samples as a function of temperature. 
Figure S10: ${ }^{7} \mathrm{Li}$ NMR spectra recorded as rotor synchronised Hahn-echoes with an increasing number of rotor periods during the echo delay.
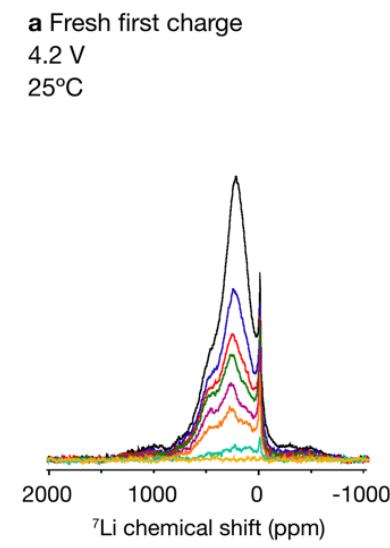

d Aged NMC811$$
28^{\circ} \mathrm{C}
$$
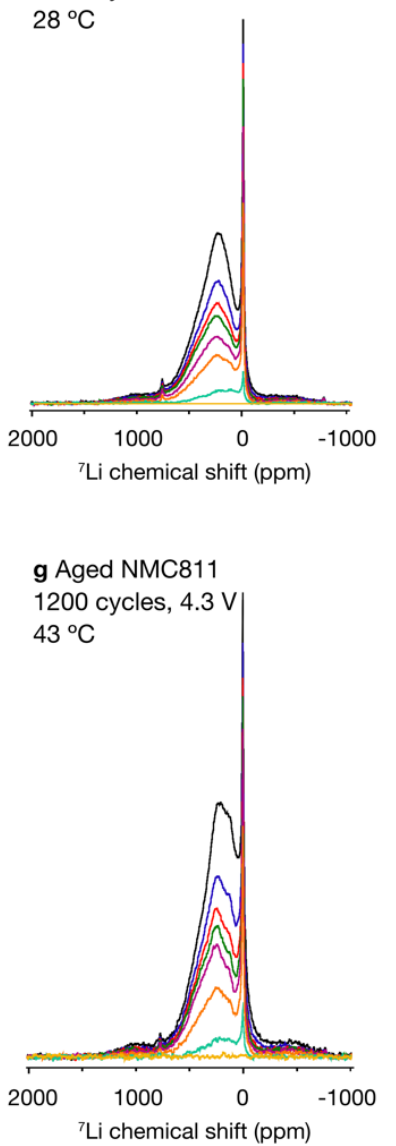
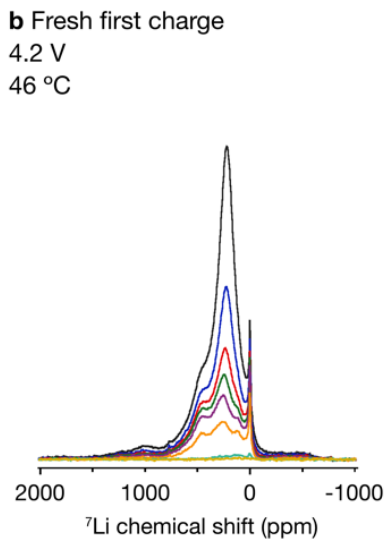

e Aged NMC811

1000 cycles, $4.2 \mathrm{~V}$

$46^{\circ} \mathrm{C}$

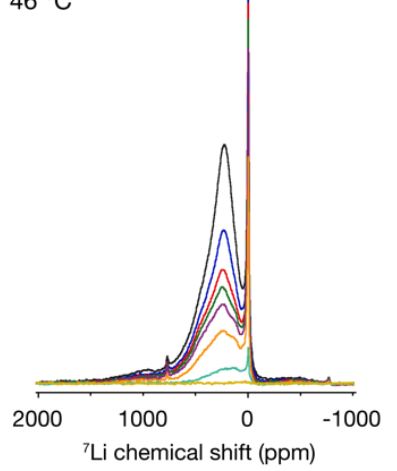

h Aged NMC811

1200 cycles, $4.3 \mathrm{~V}$

$54^{\circ} \mathrm{C}$

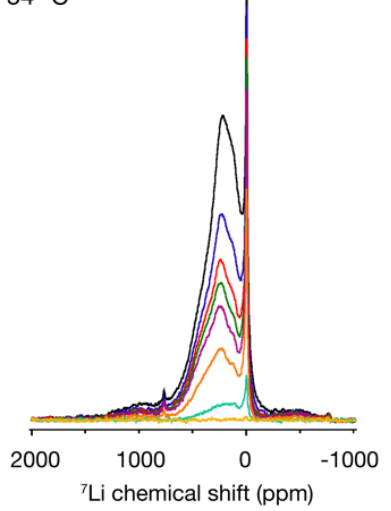

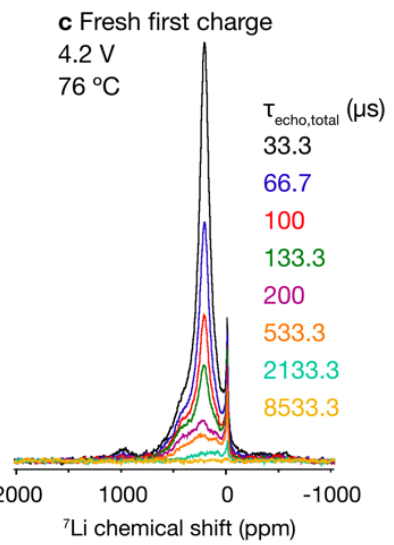

f Aged NMC811

1000 cycles, $4.2 \mathrm{~V}$

$76{ }^{\circ} \mathrm{C}$

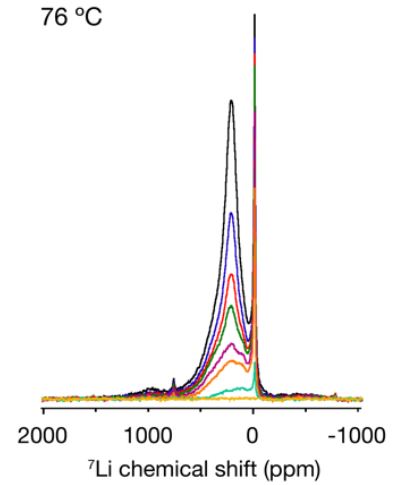

i Aged NMC811

1200 cycles, $4.3 \mathrm{~V}$

$65^{\circ} \mathrm{C}$

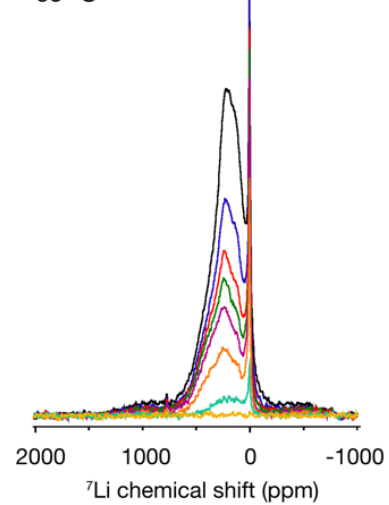

Figure S10. ${ }^{7} \mathrm{Li}$ NMR spectra recorded as rotor synchronised Hahn-echoes with an increasing number of rotor periods during the echo delay $(\tau)$ for both an NMC cathode charged to $4.2 \mathrm{~V}$ on first charge (top row), an NMC cathode after completing 1000 cycles followed by charging to and being held at $4.2 \mathrm{~V}$ (middle row) and an NMC cathode after 1200 cycles followed by charged to and being held at $4.3 \mathrm{~V}$ (bottom row). Spectra were recorded at several temperatures. The spectra where fit and $\mathrm{T}_{2} \mathrm{~S}$ calculated by fitting the data to a two-component exponential function in TopSpin. Results of this fitting are shown in Table S3 
Table S3: Spin-spin relaxation time constants $\left(\mathrm{T}_{2}\right)$ for ${ }^{7} \mathrm{Li}$ in fresh and aged NMC

Table S3. Spin-spin relaxation time constants $\left(\mathrm{T}_{2}\right)$ for ${ }^{7} \mathrm{Li}$ in $\mathrm{NMC}$ at $4.2 \mathrm{~V}$ on first charge (fresh) and after 1000 cycles and then charged to and held at $4.2 \mathrm{~V}$, and after 1200 cycles and charge to and held at $4.3 \mathrm{~V}$. The $\mathrm{I}_{0}$ of each components has been normalized to the sum the two components.

\begin{tabular}{|c|c|c|c|c|c|c|}
\hline & \multirow{2}{*}{ Temperature } & \multicolumn{2}{|c|}{ Component 1 (shorter $\left.\mathrm{T}_{2}\right)$} & \multicolumn{2}{|c|}{ Component 2 (shorter $\left.\mathrm{T}_{2}\right)$} & \multirow{2}{*}{$\mathrm{I}_{\mathrm{I}=33.3 / \mathrm{I}_{0, \text { sum }}}$} \\
\hline & & $\mathrm{T}_{2, \mathrm{comp}-1}(\mu \mathrm{s})$ & $\mathrm{I}_{0, \text { comp }-1}$ & $\mathrm{~T}_{2, \text { comp-2 }}(\mu \mathrm{s})$ & $\mathrm{I}_{0, \text { comp-2 }}$ & \\
\hline \multirow{3}{*}{$\begin{array}{l}\text { Fresh } \\
4.2 \mathrm{~V}\end{array}$} & $25^{\circ} \mathrm{C}$ & 45.3 & 0.732 & 835.9 & 0.268 & 0.610 \\
\hline & $46^{\circ} \mathrm{C}$ & 45.3 & 0.806 & 953.5 & 0.194 & 0.576 \\
\hline & $76^{\circ} \mathrm{C}$ & 53.7 & 0.855 & 996.8 & 0.145 & 0.602 \\
\hline \multirow{3}{*}{$\begin{array}{l}\text { Aged } \\
1000 \text { cycles } \\
4.2 \mathrm{~V} \text { hold }\end{array}$} & $28^{\circ} \mathrm{C}$ & 53.7 & 0.586 & 895.8 & 0.414 & 0.717 \\
\hline & $46^{\circ} \mathrm{C}$ & 48.1 & 0.695 & 1006.5 & 0.305 & 0.643 \\
\hline & $76^{\circ} \mathrm{C}$ & 53.5 & 0.766 & 948.2 & 0.234 & 0.638 \\
\hline \multirow{3}{*}{$\begin{array}{l}\text { Aged } \\
1200 \text { cycles } \\
4.3 \mathrm{~V} \text { hold }\end{array}$} & $43^{\circ} \mathrm{C}$ & 50.9 & 0.641 & 871.3 & 0.359 & 0.681 \\
\hline & $54^{\circ} \mathrm{C}$ & 50.8 & 0.645 & 877.8 & 0.355 & 0.679 \\
\hline & $65^{\circ} \mathrm{C}$ & 51.3 & 0.719 & 910.5 & 0.281 & 0.648 \\
\hline
\end{tabular}

Discussion: The $\mathrm{T}_{2}$ relaxation times for two samples are extremely similar and show a similar dependence on temperature. 


\section{Figure S11: ${ }^{7} \mathrm{Li}$ ssNMR spectra of an aged NMC cathodes after 1200 cycles at $4.3 \mathrm{~V}$ vs. Li.}

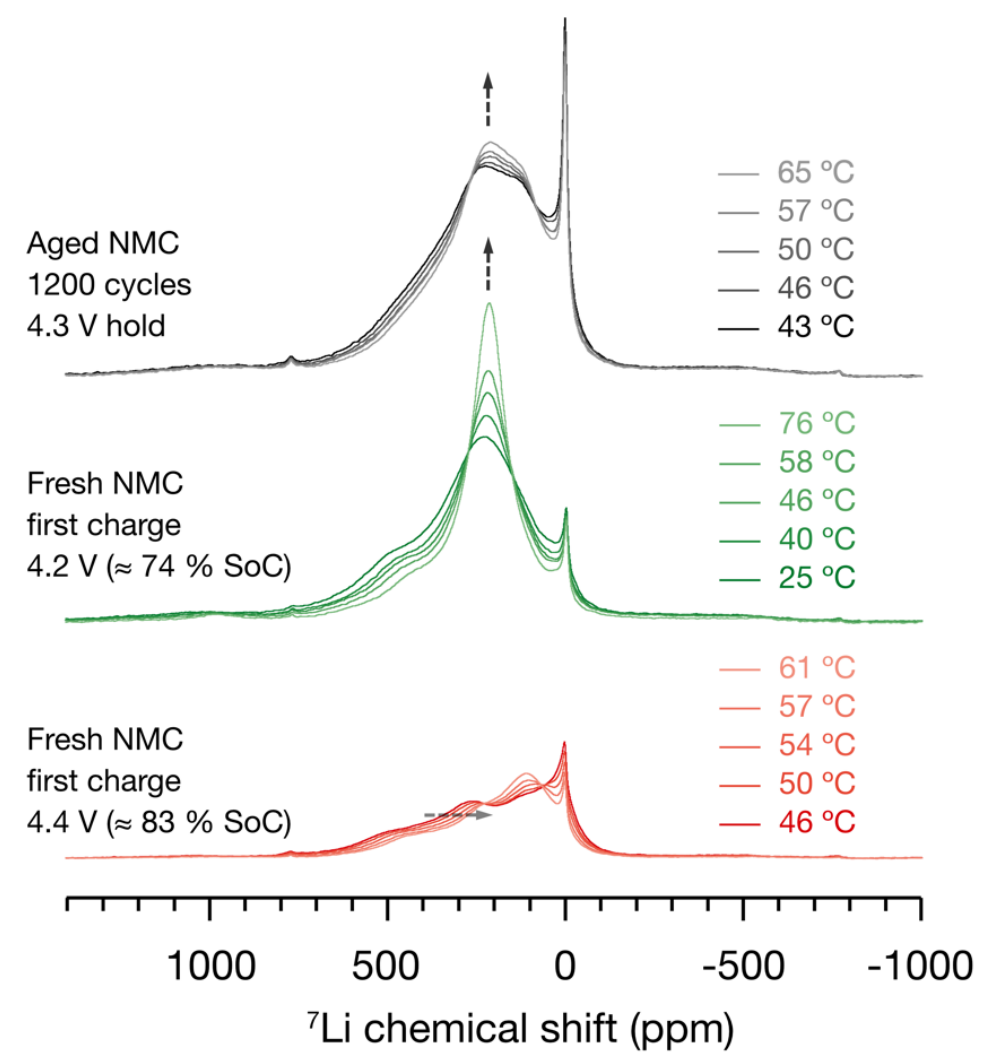

Figure S11. ${ }^{7} \mathrm{Li}$ VT ssNMR spectra of an aged NMC cathodes after 1200 cycles at $4.3 \mathrm{~V}$ vs. Li (top panel), a fresh NMC at $4.2 \mathrm{~V}$ (middle panel) and $4.4 \mathrm{~V}$ (bottom panel). The final SoC of was achieve by charging the cathode in a half cell at a rate of $\mathrm{C} / 20$ and with a voltage hold at $4.3 \mathrm{~V}$ until current drops below $\mathrm{C} / 1000$, and the corresponding diffraction analysis is shown in Figure S6. Spectra were acquired at variable temperatures at $4.7 \mathrm{~T}$ and $60 \mathrm{kHz}$ MAS frequency. The same scaling factor was applied for the spectra of each sample, and the spectra for different samples are shown with arbitrary scaling.

Discussion: Rietveld refinement of the aged NMC sample (Figure S6) shows a co- existence of fatigued particles $(\sim 76 \% \mathrm{SoC})$ and active particles $(\sim 84 \% \mathrm{SoC})$. Thereby, two fresh samples at similar SoCs, i.e. $74 \%$ and $83 \%$, were prepared and their corresponding ${ }^{7} \mathrm{Li}$ ssNMR spectra recorded at variable temperatures are presented here in Figure S11. The Li-ions in the fatigued particles is expected to have resonances at similar chemical shifts as those in the fresh sample at $4.2 \mathrm{~V}$ due to the fact they are at similar SoCs. This signal at $\sim 250 \mathrm{ppm}$ in the fresh 4.2 $\mathrm{V}$ is identified as the high-mobility Li-ions as it shows clear peak sharping upon heating for the fresh NMC sample. This effect is still observed in the aged sample, however, it is less pronounced. To verify how the active phase contributes to this, a fresh $4.4 \mathrm{~V}$ sample was measured and this resonance at $\sim 250 \mathrm{ppm}$ only shows a gradual change in the chemical shift to lower values upon heating which is attributed to the temperature-dependant nature of the Fermi-contact shift, ${ }^{10,11}$ and no obvious line-shape sharpening is observed. Therefore, the lineshape sharpening observed in the aged sample is due to the presence of high-mobility Li-ions in the fatigued phase, confirming that Li mobility is not the major origin of the fatigue degradation. 
It's also worth noting that high-mobility Li-ions are still in the $4.4 \mathrm{~V}$ sample as evidenced by the fact that the chemical shift of the peak (between 100 and $200 \mathrm{ppm}$ ) sharpens and also increases in ppm when the temperature increases. The latter is opposite to what should happen to a pure Fermi shift, and therefore is attributed to the increased mobility which leads a signal averaging over multiple Li sites at higher temperature instead. 


\section{Figure S12. (S)TEM images of the surface of an aged NMC811 particle}

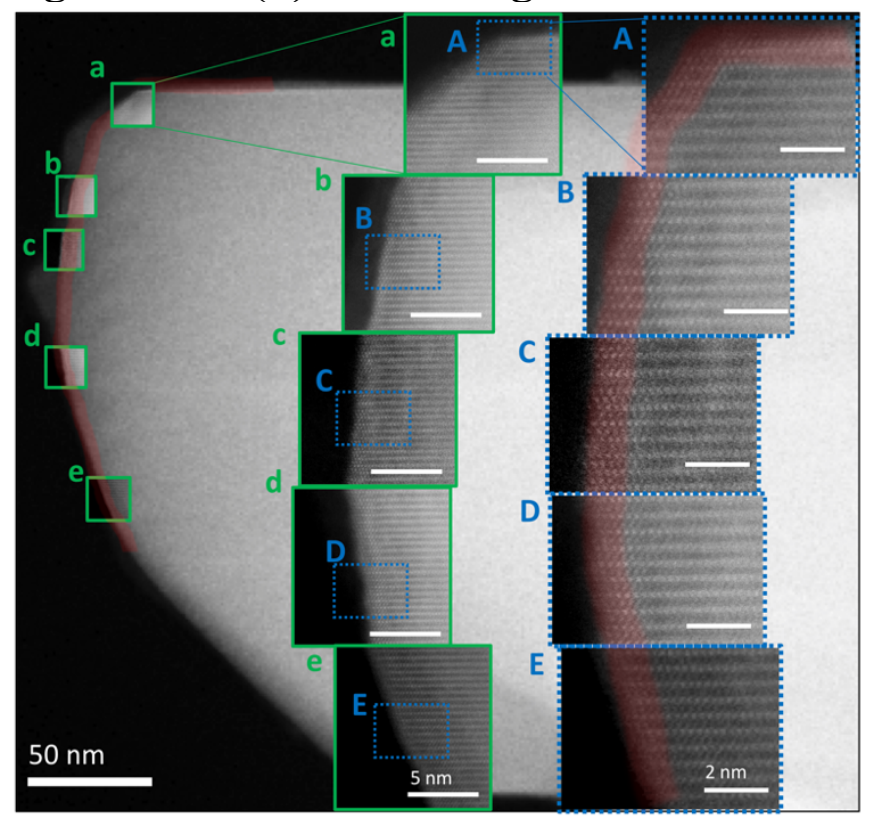

Figure S12. DF-STEM images of the surface of an aged NMC811 particle. The surface of the particle is uniformly covered by a rock salt layer. The NMC sample for STEM imaging was aged for 300 cycles, and the final SoC was at $4.3 \mathrm{~V}$ vs. Li.

Discussion: A single crystalline particle was examined by STEM. We observed that the particle was uniformly covered by a rock salt layer along the surface as highlighted in red. No rock salt structure was observed inside the particle. Several images are shown in the inset as examples. All images were taken along the $<100>$ zone axis of the layered NMC structure. 


\section{Figure S13: High resolution TEM images of the formation of surface}

reconstruction layer
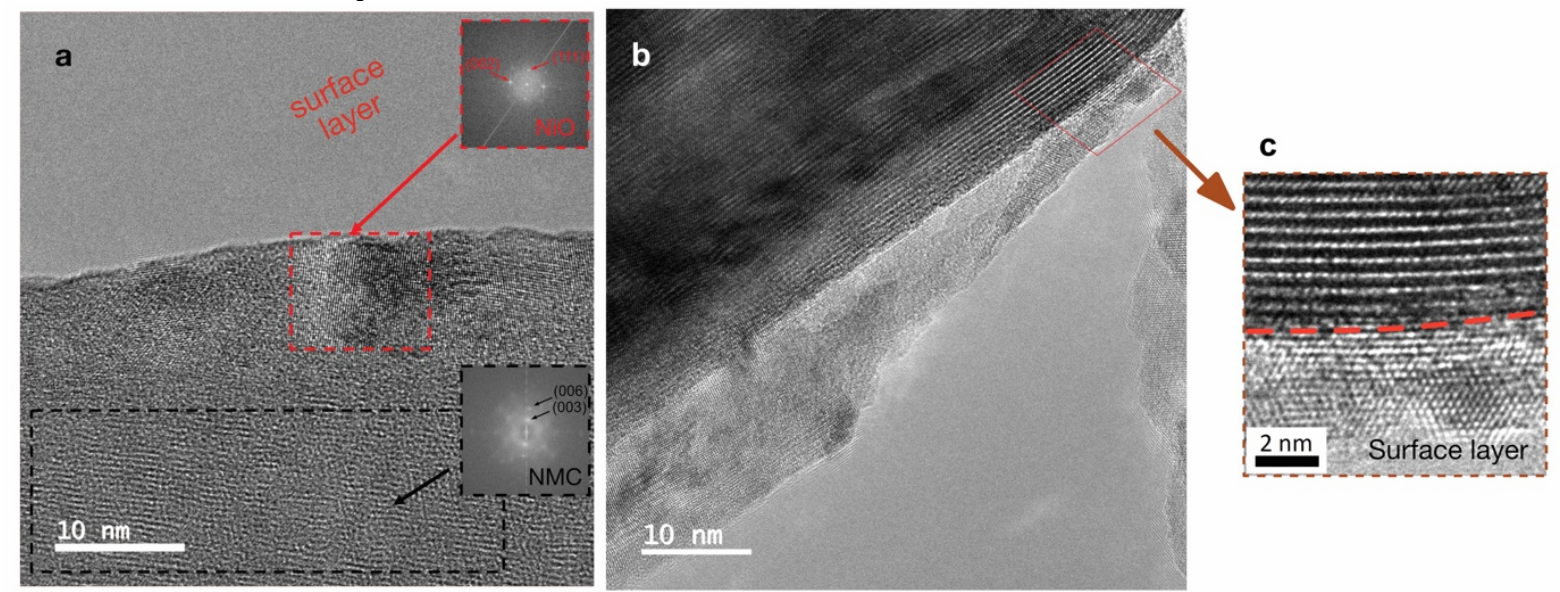

Figure S13. High-resolution TEM images of an aged NMC sample after 600 cycles and the final SoC was at $4.3 \mathrm{~V}$ vs. Li.

TEM images shown in Figure S13 were carried out using a FEI Tecnai F20 field emission gun transmission electron microscope operating at $200 \mathrm{kV}$. The TEM specimen was prepared by ion-milling the cycled NMC cathode followed by 'lift-off' of the cross-sectional lamella and subsequent attachment to a TEM grid in a FEI Helios NanoLab dual beam microscope (combining a field emission SEM and a focused ion beam). The lamella was further thinned down to render it electron transparent for TEM studies. 


\section{Figure S14: Single-crystal NMC: diffraction pattern and electrochemical performance.}
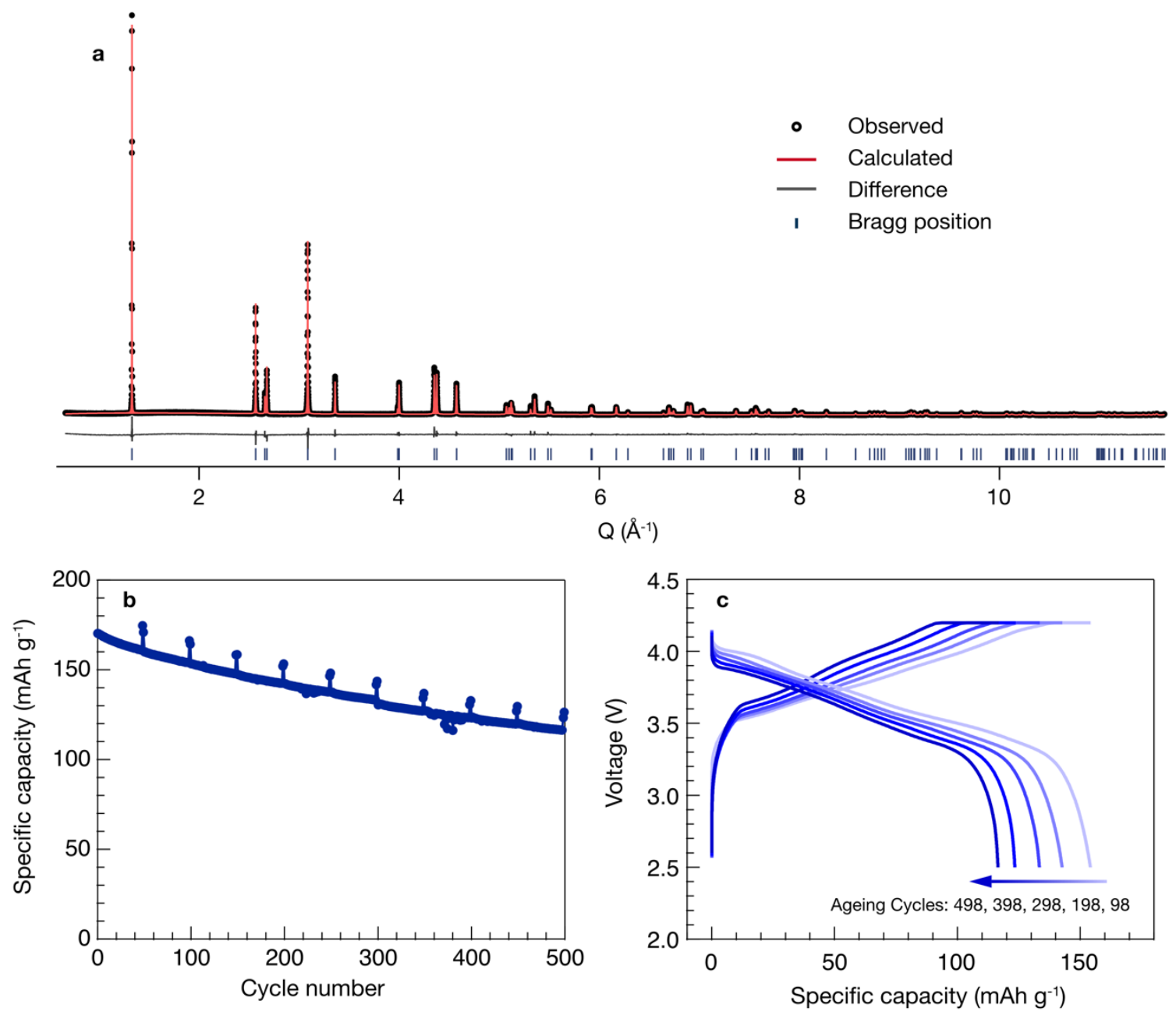

Figure S14. (a) Diffraction pattern of the single-crystal NMC. (b) Discharge capacity retention of a single-crystal NMC/graphite full cell at a rate of $\mathrm{C} / 2$ between $2.5 \mathrm{~V}$ and $4.2 \mathrm{~V}$. The electrolyte used was LP57 (1 M LiPF, EC/EMC 3/7) with 2wt\% VC. The apparent "spikes" in discharge capacity arise from the slow diagnostic cycles (C/20 rate) performed once every 50 ageing cycles. (c) Voltage profiles of a single-crystal NMC/graphite full-cell at ageing cycles of 98, 198, 298, 398 and 498. These cycles are all performed at the same rate of $\mathrm{C} / 2$. 
Table S4: Rietveld refinement result of the pristine single-crystal NMC.

Table S4. Summary of refinement results against PXRD of single-crystal NMC

\begin{tabular}{llllll}
\hline Site & Atom & $\mathrm{x}$ & $\mathrm{y}$ & $\mathrm{z}$ & Occupancy \\
\hline $3 \mathrm{a}$ & $\mathrm{Ni}$ & 0 & 0 & 0 & $0.8160(9)$ \\
& $\mathrm{Mn}$ & 0 & 0 & 0 & 0.1 \\
& $\mathrm{Co}$ & 0 & 0 & 0 & 0.06 \\
& $\mathrm{Li}$ & 0 & 0 & 0 & $0.0240(9)$ \\
$3 \mathrm{~b}$ & $\mathrm{Li}$ & 0 & 0 & $1 / 2$ & $0.9760(9)$ \\
& $\mathrm{Ni}$ & 0 & 0 & $1 / 2$ & $0.0240(9)$ \\
$6 \mathrm{c}$ & $\mathrm{O}$ & 0 & 0 & $0.25871(9)$ & 1 \\
\hline
\end{tabular}

Space group: $R \overline{3} m$

Fitting quality: $\mathrm{R}_{\mathrm{wp}}=11.240, \mathrm{R}_{\exp }=3.203$, goodness of fit $=3.509$

Lattice parameters: $a=b=2.87265(1) \AA, c=14.19168(8) \AA$, unit cell volume $=$ 101.4213(8) $\AA^{3}$

The nominal transmission metal stoichiometry Ni:Mn:Co is constrained to be $0.84: 0.06: 0.1$ as determined by inductively coupled plasma atomic emission spectroscopy (ICP-OES) and Li occupancy is 1 . 
Figure S15: X-ray diffraction pattern of aged single-crystal NMC at a charged state.

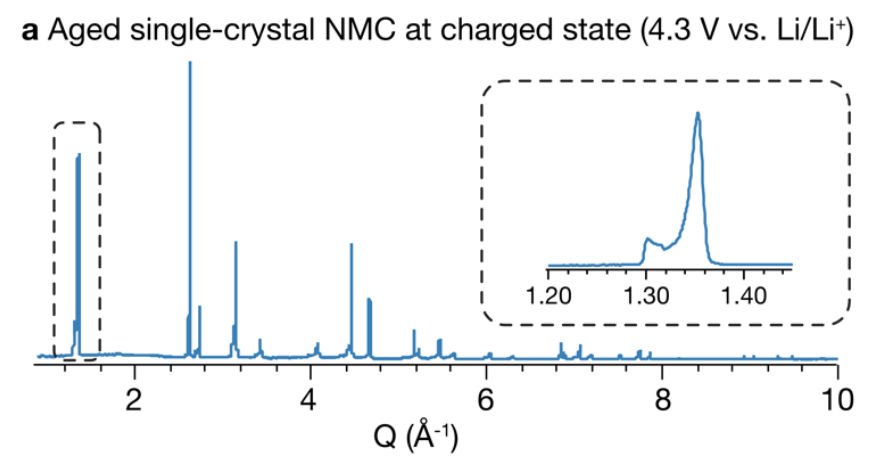

b Aged single-crystal NMC at charged state $\left(4.6 \mathrm{~V}\right.$ vs. $\left.\mathrm{Li}^{\mathrm{L}} \mathrm{Li}^{+}\right)$

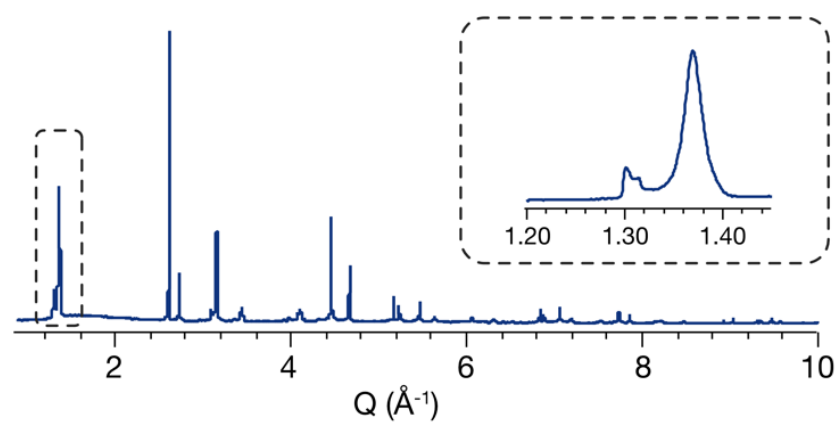

Figure S15. Diffraction pattern of electrochemically aged NMC at (a) $4.3 \mathrm{~V}$ and (b) $4.6 \mathrm{~V}$ after 500 cycles. 
Figure S16. Analysis of the do03 spacing for the pristine and aged NMC811 particles.
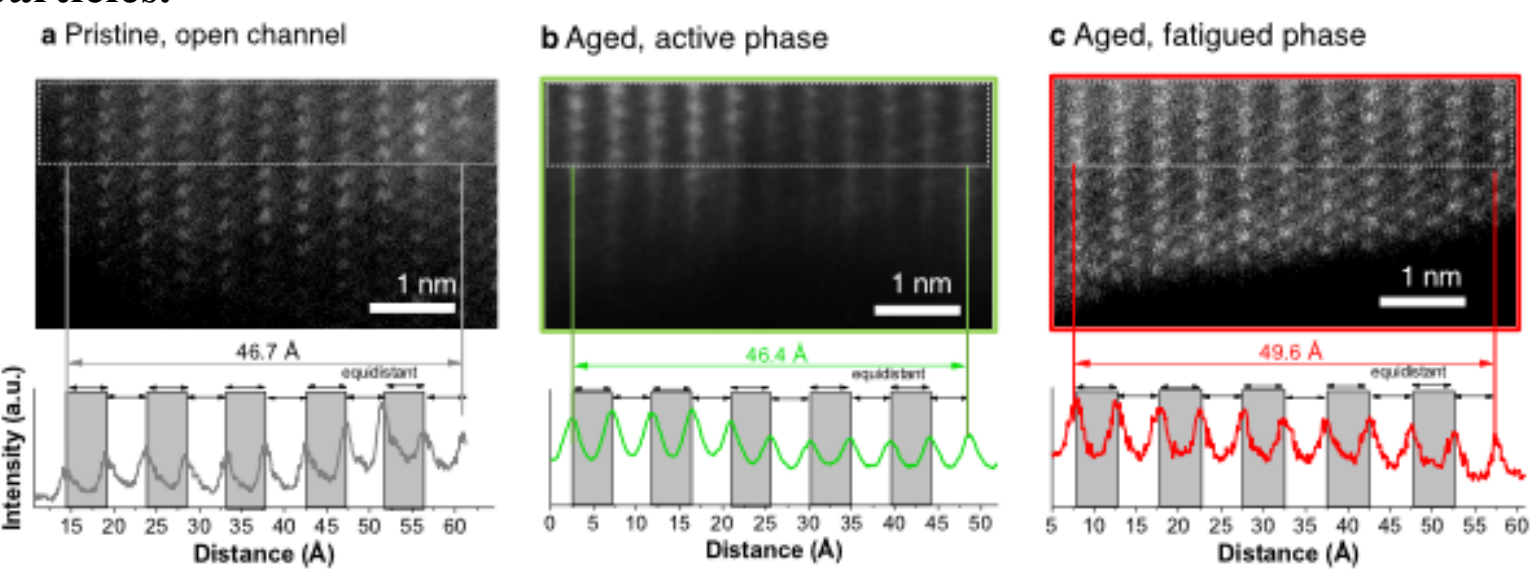

Figure S16. Image analysis of $\mathrm{d}_{003}$ spacing for the pristine (a), and the aged NMC particles with open lithium transportation channel (b) and the rock salt surface reconstruction layer (c). For the analysis, the distance of 10 layers was measured.

Discussion: For measuring the distance of ten consecutive layers of the (003) plane, signal intensity profiles were obtained by Gatan Microscopy Suite from the dotted box in each image. Then, the distance was measured by reading the peak intensities. The equidistant between the layers indicates the negligible level of sample drift during image acquisition. Another source of error, i.e. sample tilt, will lead to a decrease in the measured layer spacing as compared to the actual value. We simulated a scenario (based on a trigonometry calculation) that a sample title of nearly $16.5^{\circ}(290 \mathrm{mrad})$ is required to change to the layer spacing from $50 \AA$ to $\sim 47 \AA$. With such large tilting degrees from the zone axis, the individual atomic columns will be significantly misaligned and cannot be resolved on the image. Hence, it is less likely that the net difference of $3 \AA$ was caused by the sample tilt error. 


\section{References}

1. Radin, M. D. et al. Narrowing the Gap between Theoretical and Practical Capacities in Li-Ion Layered Oxide Cathode Materials. Adv. Energy Mater. 7, 1602888 (2017).

2. Li, H., Zhang, N., Li, J. \& Dahn, J. R. Updating the Structure and Electrochemistry of $\mathrm{Li}_{x} \mathrm{NiO}_{2}$ for $0 \leq \mathrm{x} \leq 1$. J. Electrochem. Soc. 165, A2985-A2993 (2018).

3. Wang, L., Maxisch, T. \& Ceder, G. A First-Principles Approach to Studying the Thermal Stability of Oxide Cathode Materials. Chem. Mater. 19, 543-552 (2007).

4. Kleiner, K. et al. Fatigue of $\mathrm{LiNi}_{0.8} \mathrm{Co}_{0.15} \mathrm{Al}_{0.05} \mathrm{O}_{2}$ in commercial Li ion batteries. J. Power Sources 273, 70-82 (2015).

5. Liu, H. et al. Intergranular Cracking as a Major Cause of Long-Term Capacity Fading of Layered Cathodes. Nano Lett 17, 3452-3457 (2017).

6. Friedrich, F. et al. Editors' Choice-Capacity Fading Mechanisms of NCM-811 Cathodes in Lithium-Ion Batteries Studied by X-ray Diffraction and Other Diagnostics. J. Electrochem. Soc. 166, A3760-A3774 (2019).

7. Schweidler, S. et al. Investigation into Mechanical Degradation and Fatigue of High-Ni NCM Cathode Material: A Long-Term Cycling Study of Full Cells. ACS Applied Energy Materials 2, 7375-7384 (2019).

8. Märker, K., Reeves, P. J., Xu, C., Griffith, K. J. \& Grey, C. P. Evolution of Structure and Lithium Dynamics in $\mathrm{LiNi}_{0.8} \mathrm{Mn}_{0.1} \mathrm{Co}_{0.1} \mathrm{O}_{2}(\mathrm{NMC} 811)$ Cathodes during Electrochemical Cycling. Chem. Mater. 31, 2545-2554 (2019).

9. Norris, J. R. Rapid computation of magnetic resonance line shapes for exchange among many sites. Chem. Phys. Lett. 1, 333-334 (1967).

10. Carlier, D., Ménétrier, M., Grey, C. P., Delmas, C. \& Ceder, G. Understanding the NMR shifts in paramagnetic transition metal oxides using density functional theory calculations. Physical Review B 67, 174103 (2003).

11. Grey, C. P. \& Dupré, N. NMR Studies of Cathode Materials for Lithium-Ion Rechargeable Batteries. Chem. Rev. 104, 4493-4512 (2004). 